\title{
Braid Group Representations from Braiding Gapped Boundaries of
} Dijkgraaf-Witten Theories

\author{
by \\ Nicolás Escobar Velásquez \\ A dissertation submitted in partial satisfaction of the \\ requirements for the degree of \\ Master in Science \\ in \\ Mathematics \\ of \\ Universidad de los Andes
}

Advisor:

César Neyit Galindo Martinez

2017 


\begin{abstract}
Braid Group Representations from Braiding Gapped Boundaries of Dijkgraaf-Witten Theories

by

Nicolás Escobar Velásquez

Master in Science in Mathematics

Universidad de los Andes

Advisor: César Neyit Galindo Martinez

In Topological Quantum Computation, quantum gates are implemented by representations of the braid group, $\mathcal{B}_{n}$, on spaces of morphisms in a modular category $\mathcal{C}$. For a given group $G$ and a 3 -cocycle $\omega$, images of that representation on $\mathcal{C}=\mathcal{Z}\left(\operatorname{Vec}_{G}^{\omega}\right)$ are finite, but is not known in general what specific gates can be obtained. A family of algebras in $\mathcal{Z}(G, \omega)$ called Lagrangian Algebras are of particular physical interest. They are denoted $L[H, \gamma]$, where $H$ is a subgroup and $\gamma$ is a 2-cocycle on $H$. We show that the spaces $\operatorname{Hom}_{\mathcal{Z}(G, \omega)}\left(\mathbb{1}, L[H, \gamma]^{\otimes n}\right)$ have a canonical structure of monomial spaces and that with respect to this structure, the representation of $\mathcal{B}_{n}$ is monomial. We calculate the nonzero entries of these matrices and use this information to show how they can be used to implement a CNOT gate.
\end{abstract}


Para Camilo, Ma y Pa. 


\section{Contents}

Contents

1 Introduction $\quad 1$

2 Braid group representations $\quad 3$

2.1 Monoidal Categories . . . . . . . . . . . . . . . . . . . . 3

2.2 Braided Categories . . . . . . . . . . . . . . . . . . . . 6

2.3 Lagrangian Algebras . . . . . . . . . . . . . . . . . . . . . . . . . . 10

3 Monomiality of $\psi_{L[H, \gamma]} \quad 12$

3.1 Monomial representations . . . . . . . . . . . . . . . . . . 12

3.2 Monomial representation of the braid group . . . . . . . . . . . . . 14

3.3 Braid groups representations associated to Lagrangian algebras . . . . . . . . 17

4 Applications and examples 21

4.1 Computing dimensions of ground states . . . . . . . . . . . . . . . . . . 21

4.2 Quantum Gates . . . . . . . . . . . . . . . . . . 24

$\begin{array}{lr}\text { Bibliography } & 29\end{array}$ 


\section{Acknowledgments}

I want to thank professor César Galindo. A couple of years ago he organized the first Colombian School of Quantum Computation, which got me interested in the subject. He has thought me everything I know about categories. He provided me with powerful insights about representations of the braid group. And he gave me guidance, patience and counsel that I will always appreciate.

Special thanks are due to professor Zhenghan Wang. He suggested this research topic, helped writing a paper associated to this project and attended many questions we had throughout this process.

Aknowledgments also go to professor Eric Rowell, who invited me to present this project to his research team and gave me valuable feedback.

I am also in debt with other members of the research group on Category Theory at Universidad de los Andes: César Venegas, Nicolás Ramirez and Nicolás Jaramillo. Their talks at the group seminar were very useful to me, as well as our informal discussions.

Finally, a note of gratitude to the Mathematics Department and the Faculty of Science at Universidad de los Andes. Their support has been overwhelming. I could not ask more from my alma mater.

On a more personal note, I would like to thank professors Andrés Reyes, Andrés Ángel and Monika Winklmeier. Their advice and encouragment througout these years at Universidad de los Andes has been invaluable. 


\section{Chapter 1}

\section{Introduction}

Quantum Computation can be roughly defined as the use of the laws of quantum Mechanics to implement information processing.

It started as an idea by David Deutsch to challenge the Church-Turing hyphotesis [7]. If no classical computer design seemed to be more powerful than a Turing machine, then maybe one that used a quantum system to store and manipulate information could.

On the other hand, Feynman noticed that classical computers are not efficient simulating quantum systems [12]. This is an important difficulty because problems as the interactions between molecules and membranes of cells, while governed by the laws of quantum mechanics, are too complicated to be solved analytically. However, some quantum systems can act like universal computers, in the sense that they can simulate other, more complex ones. Thus, even if quantum computers could not solve more problems than a Turing machine, they could solve of them some faster.

This idea got reinforced by the work of Shor. He showed that a quantum computer could find the prime factors of a number in polynomial time [20], something classical computers are thought to be unable to do. Because of the implications of this discovery to cryptography, this generated a lot of attention. Something similar happens with the problem of finding elements in a list, for which quantum computers provide a quadratic speedup [13]. However, it should be noted that the range of problems for which quantum computers are faster than classical ones is limited.

These theoretical results raised the question of how to build and actual quantum computer. A major difficulty here is quantum decoherence. Roughly speaking, quantum systems interact strongly with the surrounding environment. This introduces noise and generates errors. Classical computers also experience errors of this kind, but they can be corrected easily. Quantum error correction is more difficult and is an area of active research. Most paradigms for the implementation of quantum computers have to deal with error correction one way or the other. A completely different approach to the problem of quantum decoherence is to create quantum systems that are fault tolerant. This means that while they are subject to errors, these do not affect the storing of information or the outcome of computations.

This is were Kitaev's work comes into play. In a seminal paper [17], he did two things of 
interest. First, he gave a Hamiltonian realization of Dijkgraaft-Witten TQFT. That means that given a closed surface, he constructed a Hilbert space and a Hermitian operator on it, whose eigenspace with the lowest eigenvalue turns out to be the vector space associated to that surface by the Dijkgraaft-Witten TQFT. We call this vector space the ground state of the system. Second, and more importantly, he realized that this subspace is preserved by the action of local errors and therefore is ideal to store quantum information.

To make matters even more interesting, this system is completely solvable. Completely solvable models are hard to find and are something of a holy grail because they provide important insight into models that are not. Explicit solutions of this model show that it has a collection of elementary excitations or quasiparticles. This quasiparticles can fuse together to create new excitations. Moreover, they pick up phases when rounded around each other. The behavior of such quasiparticles is modeled by a modular category, called the topological order of the model. In this case, that modular category turns out to be the category of representations of the Drinfeld double of the gauge group of the model, $\operatorname{Rep}(D(G))$.

There is a problem though. The amount of information that can be stored in a quantum system is proportional to the dimension of the underlying Hilbert space. In Kitaev's case, this dimension is called the degeneracy. In Kitaev's construction, the degeneracy is related to the genus of the surface. This presents a difficulty because, while two dimensional quantum systems are not that hard to construct nowadays, only plane surfaces can be built in the laboratory. Thus, a scalable design (one that be carried over to store as much information as needed) was not feasible in this paradigm.

A solution to this problem came by studying extensions of the Hamiltonian realization of the Dijkgraaft-Witten TQFT to surfaces with boundary. In [4, 3, 2], Bravyi, Kitaev, Beigi, Bombin and Martin Delgado found that boundaries increased the the degeneracy. By considering a plane with arbitrary number of holes, we can create numerous boundaries and hence make the degeneracy as large as necessary. Moreover, it was realized that these boundaries were modeled by Lagrangian algebras in the topological order of the system.

Finally, in [5] Cong, Cheng and Wang set up a way to use Lagrangian algebras to implement quantum operations. Specifically, braidings of these algebras generate a representation of the braid group, whose images are quantum gates. However, questions about the images of this representations were left open. Answering some of these questions is our main goal.

This document is organized as follows. Chapter 2 presents some basic results about monoidal and braided categories, as well as the braid group and Lagrangian algebras. Chapter 3 contains the main results of this work. Specifically, it introduces the notion of monomial representations and monomial Yetter-Drinfeld modules. Then, using a specific basis, it is shown that Lagrangian algebras are an example of this last kind of module and how this implies that the representation of $\mathcal{B}_{n}$ in an associated Hom space is monomial. Chapter 4 shows some applications of these ideas to the computation of degeneracies and the implementation of specific quantum gates.

Results in Chapter 3 and Section 4.1 have been submited for publication. A preprint can be found in arXiv:1707.03884 [8]. The designs for the implementation of qubits and the gate CNOT found in Section 4.2 appear here for the first time. 


\section{Chapter 2}

\section{Braid Group Representations from Braided Categories}

The basic mathematical structure underlying this document is that of a modular category. Modular categories have an increasingly important role in solid state physics and in the last couple of decades, they have found applications in Topological Quantum Computation. We will not use the full power of these categories. To us, braided categories will be enough. In this chapter we define them and present some basic results. We will assume the reader is familiar with the basic concepts of category theory, an account of which can be found in [18]. For a detailed exposition on modular categories, see [11, 21, 1].

Throughout this document, $\mathbb{C}$ denotes the field of complex numbers. For simplicity, we work only with vector spaces over $\mathbb{C}$, unless otherwise specified. However, most results can be generalized to other fields.

\subsection{Monoidal Categories}

Definition 2.1.1. A monomial category is a tuple $(\mathcal{C}, \otimes, a, \mathbb{1}, l, r)$. Here $\mathcal{C}$ is a category, $\otimes$ is a bifunctor $\otimes: \mathcal{C} \times \mathcal{C} \rightarrow \mathcal{C}$ called the tensor product and $a$ is the associativity constraint, that is: a natural isomorphism

$$
a_{X, Y, Z}:(X \otimes Y) \otimes Z \rightarrow X \otimes(Y \otimes Z) \quad X, Y, Z \in \operatorname{Obj}(\mathcal{C})
$$

such that the diagram

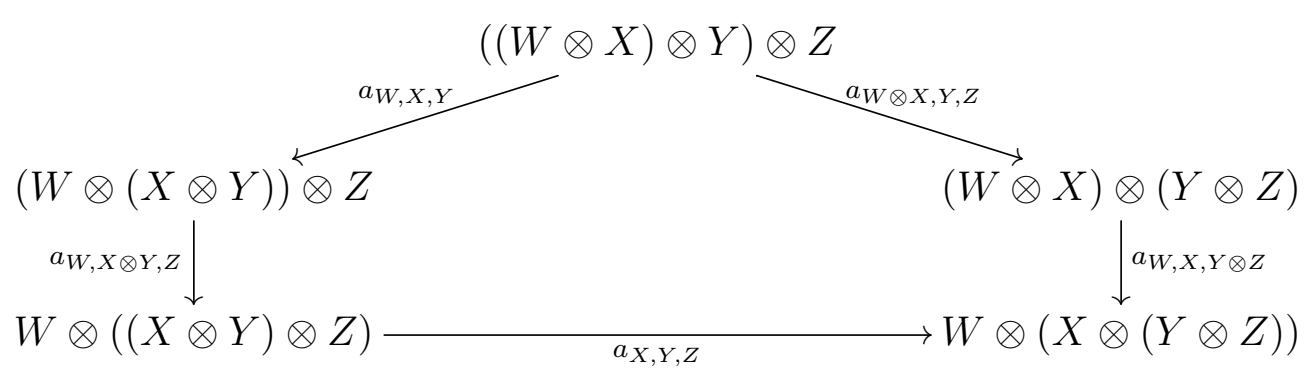


commutes for all objects $X, Y, Z, W \in \mathcal{C}$. The last components of the tuple, $l$ and $r$, are natural isomorphisms $l_{X}: \mathbb{1} \otimes X \rightarrow X$ and $r_{X}: X \otimes \mathbb{1} \rightarrow X$ for which the diagrams

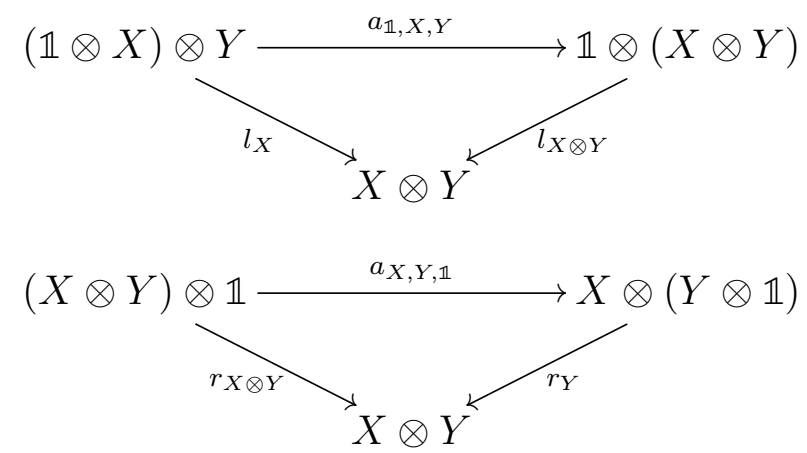

commute for all objects $X, Y$ of $\mathcal{C}$. We call $\mathbb{1}$ the unit element and isomorphisms $l_{x}$ and $r_{x}$ the unit constraints.

Example 2.1.1. Let $G$ be a finite group. Let $Z^{3}\left(G, \mathbb{C}^{\times}\right)$be the set of functions $\omega: G \times G \times$ $G \rightarrow \mathbb{C}^{\times}$such that

$$
\omega\left(g_{1} g_{2}, g_{3}, g_{4}\right) \omega\left(g_{1}, g_{2}, g_{3} g_{4}\right)=\omega\left(g_{1}, g_{2}, g_{3}\right) \omega\left(g_{1}, g_{2} g_{3}, g_{4}\right) \omega\left(g_{2}, g_{3}, g_{4}\right)
$$

Fix an $\omega \in Z^{3}\left(G, \mathbb{C}^{\times}\right)$. We define $\operatorname{Vec}_{G}^{\omega}$ as the category whose objects are finite dimensional vector spaces $V$ with a family of subspaces $\left\{V_{g}\right\}_{g \in G}$ such that $V=\bigoplus_{g \in G} V_{g}$. These subspaces are called components and the decomposition, a $G$-grading. Vectors contained in one of the components are called $G$-homogeneous. For a $G$ homogeneous vector $v$ and $g \in G$, we write $|v|=g$ if $v \in V_{g}$. We often refer to $V_{e}$ as the component in trivial degree. A morphism between objects $V=\bigoplus_{g \in G} V_{g}$ and $W=\bigoplus_{g \in G} W_{g}$ is a linear map $T: V \rightarrow W$ such that $T\left(V_{g}\right)=W_{g}$ for each $g \in G$. We refer to this last condition as preserving the grading.

The bifunctor $\otimes$ is the ordinary tensor product of vector spaces. For objects $V=\bigoplus_{g \in G} V_{g}$ and $W=\bigoplus_{g \in G} W_{g}$,

$$
(V \otimes W)_{g}=\bigoplus_{h \in G} V_{h} \otimes W_{h^{-1} g} \quad g \in G
$$

The unit object consists of a copy of $\mathbb{C}$ in the component of trivial degree. The unit constraints are

$$
\begin{aligned}
r_{\delta_{g}}: \mathbb{1} \otimes \delta_{g} & \rightarrow \delta_{g}=\omega(g, 1,1) \operatorname{Id}_{\delta_{g}} \\
l_{\delta_{g}}: \delta_{g} \otimes \mathbb{1} & \rightarrow \delta_{g}=\omega(1,1, g)^{-1} \operatorname{Id}_{\delta_{g}}
\end{aligned}
$$

The associativity constraint, restricted to components, is

$$
a_{U, V, W}=\left(U_{g} \otimes V_{h}\right) \otimes W_{k} \rightarrow U_{g} \otimes\left(V_{h} \otimes W_{k}\right): \omega(g, h, k) a_{\left(U_{g} \otimes V_{h}\right) \otimes W_{k}}^{\mathrm{Vec}}
$$

where $a^{\mathrm{Vec}}$ is the natural isomorphism that defines the associativity in the category of vector spaces. 
Definition 2.1.2. Let $(\mathcal{C}, \otimes, \mathbb{1}, a, l, r)$ and $\left(\mathcal{C}^{\prime}, \otimes^{\prime}, \mathbb{1}^{\prime}, a^{\prime}, l^{\prime}, r^{\prime}\right)$ be monoidal categories. A monoidal functor from $\mathcal{C}$ to $\mathcal{C}^{\prime}$ is a triple $(F, J, \varphi)$ where $F: \mathcal{C} \rightarrow \mathcal{C}$ is a functor, $J$ is a natural isomorphism

$$
J_{X, Y}: F(X) \otimes F(Y) \rightarrow F(X \otimes Y)
$$

such that

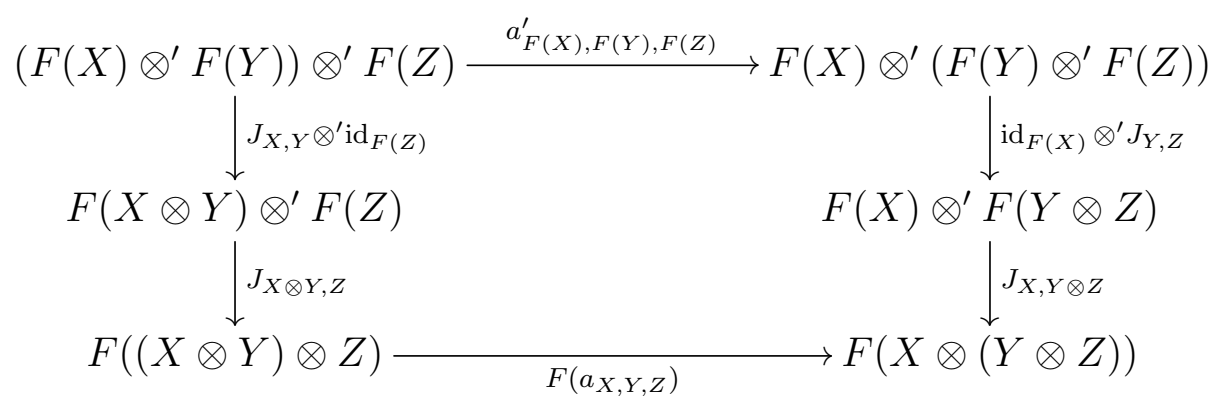

commutes for all objects $X, Y, Z$ of $\mathcal{C}$ and the map $\varphi$ is an isomorphism $\varphi: \mathbb{1}^{\prime} \rightarrow F(\mathbb{1})$ with the property that

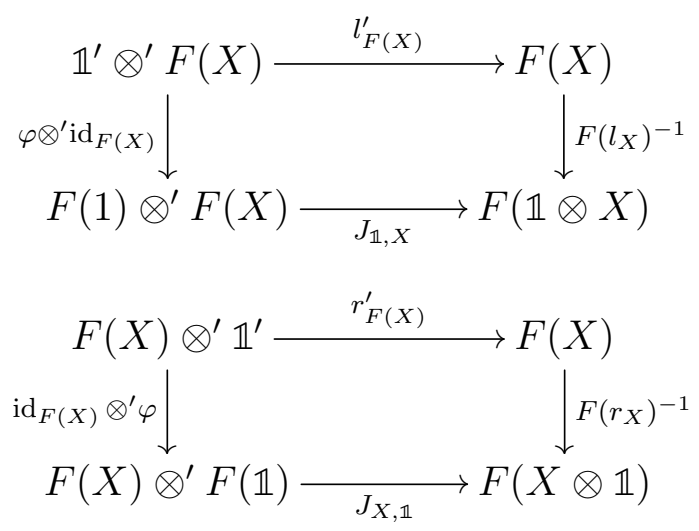

commute for all objects $X$ of $\mathcal{C}$. A monoidal equivalence is a monoidal functor $(F, J, \varphi)$ where $F$ is an equivalence of categories.

Remark 2.1.1. Definition 2.1.2 actually corresponds to what is known in the literature as a strong monoidal functor. The usual definition of a monoidal functor does not require the maps $J$ and $\varphi$ to be isomorphisms. However, we will only encounter strong monoidal functors in this document, which justifies our abuse in terminology.

Remark 2.1.2. A monoidal category is called strict if $(X \otimes Y) \otimes Z=X \otimes(Y \otimes Z)$ and $a_{X, Y, Z}=\operatorname{id}_{(X \otimes Y) \otimes Z}$ rather than just being naturally isomorphic. By Mac Lane's strictness theorem [18] every monoidal category is equivalent to a strict one.

Definition 2.1.3. Let $\mu: G \times G \rightarrow \mathbb{C}^{\times}$be any function. We define a new function $d^{3} \mu$ : $G \times G \times G \rightarrow \mathbb{C}^{\times}$by

$$
d^{3} \mu\left(g_{1}, g_{2}, g_{3}\right)=\frac{\mu\left(g_{2}, g_{3}\right) \mu\left(g_{1}, g_{2} g_{3}\right)}{\mu\left(g_{1} g_{2}, g_{3}\right) \mu\left(g_{1}, g_{2}\right)} \quad g_{1}, g_{2}, g_{3} \in G
$$


Two functions $\omega, \omega^{\prime}: G \times G \times G \rightarrow \mathbb{C}^{\times}$are said to be cohomologous if there exists a function $\mu: G \times G \rightarrow \mathbb{C}^{\times}$such that

$$
\omega=\omega^{\prime} d^{3} \mu
$$

Proposition 2.1.1. Let $G$ be a group and $\omega_{1}, \omega_{2} \in Z^{3}\left(G, \mathbb{C}^{\times}\right)$two 3-cocycles. Then $\operatorname{Vec}_{G}^{\omega_{1}}$ and $\operatorname{Vec}_{G}^{\omega_{2}}$ are monoidally equivalent if and only if $\omega_{1}$ and $\omega_{2}$ are cohomologous.

Proof. If $\omega_{1}, \omega_{2}$ are cohomologous, let $\mu: G \times G \rightarrow \mathbb{C}^{\times}$such that

$$
\omega_{1}=\omega_{2} d_{3}(\mu) \text {. }
$$

Let $F=\operatorname{Id}_{\operatorname{Vec}_{g}^{\omega_{1}}}$ and define the natural transformation

$$
J_{g, h}=\mu(g, h) \operatorname{id}_{\delta_{g h}}: F\left(\delta_{g}\right) \otimes F\left(\delta_{h}\right) \rightarrow F\left(\delta_{g h}\right) .
$$

Condition (2.1) reduces to (2.2). Details can be found in [9, section 2.6].

Remark 2.1.3. In the definition of $\operatorname{Vec}_{G}^{\omega}$, we may assume that $\omega$ is normalized, in the sense that $\omega(g, 1, h)=1$ for all $g, h \in G$. Indeed, if necessary we can replace $\omega$ by $\omega d_{3}(\mu)$ where $\mu$ is any 2-cochain such that $\mu(g, 1)=\omega(g, 1,1)$ and $\mu(1, h)=\omega(1,1, h)^{-1}$. See [9, Remark 2.6.3] for more details. In particular, for all $g \in G, l_{\delta_{g}}=r_{\delta_{g}}=\mathrm{id}_{\delta_{g}}$.

\subsection{Braided Categories}

Definition 2.2.1. A braiding on a monoidal category $\mathcal{C}$ is a natural isomorphism

$$
c_{X, Y}: X \otimes Y \rightarrow Y \otimes X \quad X, Y \in \mathcal{C}
$$

such that the diagrams

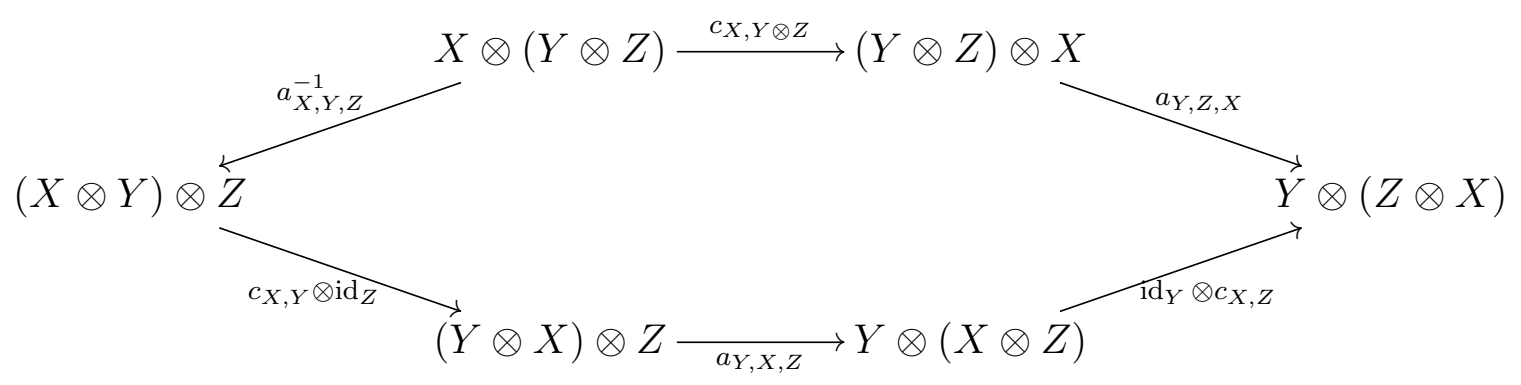

and

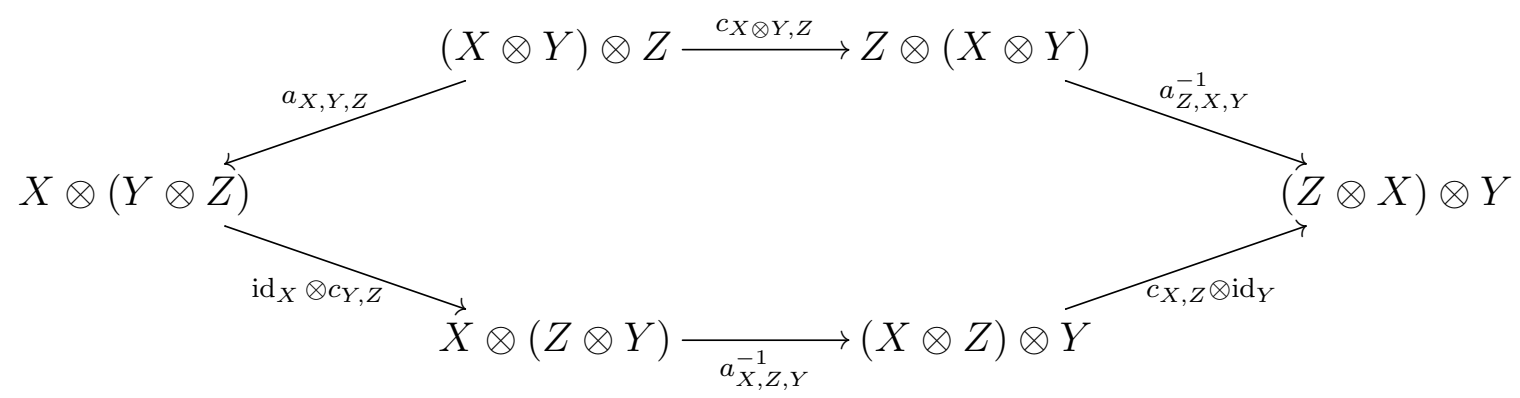


commute. A monoidal category with a braiding is called a braided category [14].

We now define the Drinfeld center of a monoidal category. It allows us to construct a braided category starting from a monoidal one. In a sense, a monoidal category is the categorification of a monoid. The Drinfeld center corresponds to taking the center of that module. Here is the formal definition.

Definition 2.2.2. Let $(\mathcal{C}, \otimes, \mathbb{1}, a, l, r)$ be a monoidal category. Its Drinfeld center, $\mathcal{Z}(\mathcal{C})$, is the braided monoidal category defined as follows. Objects are pairs $(Z, \gamma)$ where $Z$ is an object of $\mathcal{C}$ and $\gamma_{X}: X \otimes Z \rightarrow Z \otimes X$ are natural isomorphisms for which the diagram

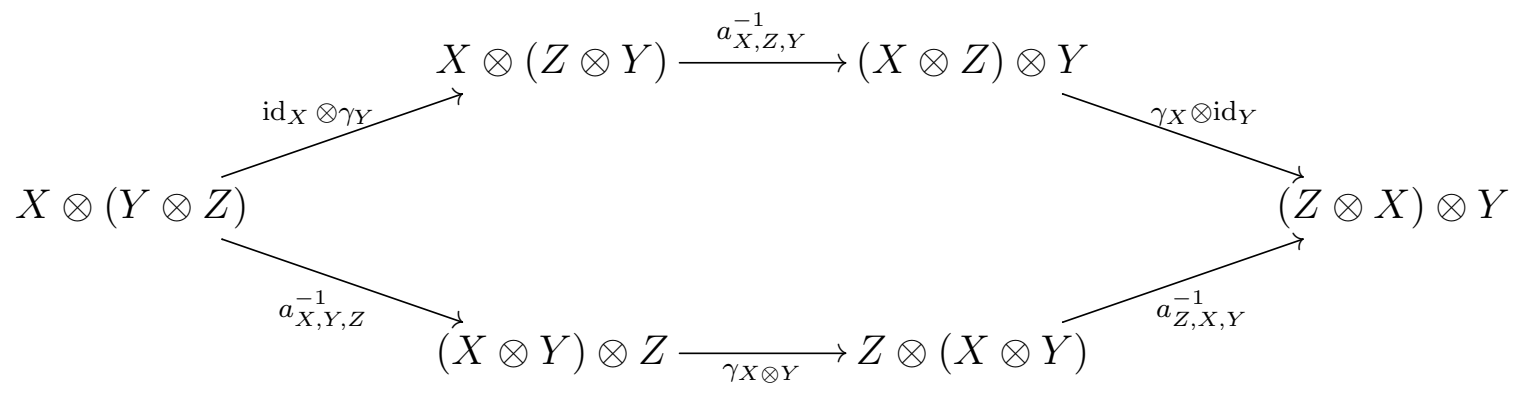

commutes for all objects $X, Y$ of $\mathcal{C}$. Morphisms between $(Z, \gamma)$ and $\left(Z^{\prime}, \gamma^{\prime}\right)$ are morphisms $f \in \operatorname{Hom}_{\mathcal{C}}\left(Z, Z^{\prime}\right)$ for which

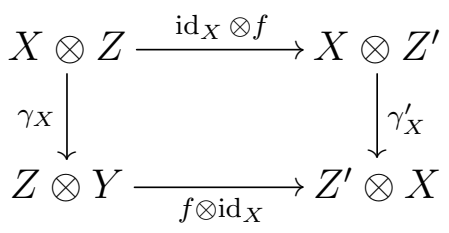

commutes for all $X \in \operatorname{Obj}(\mathcal{C})$.

This is a monoidal category with tensor product

$$
(Z, \gamma) \otimes\left(Z^{\prime}, \gamma^{\prime}\right)=\left(Z \otimes Z^{\prime}, \tilde{\gamma}\right)
$$

where $\tilde{\gamma}$ is defined by the diagram

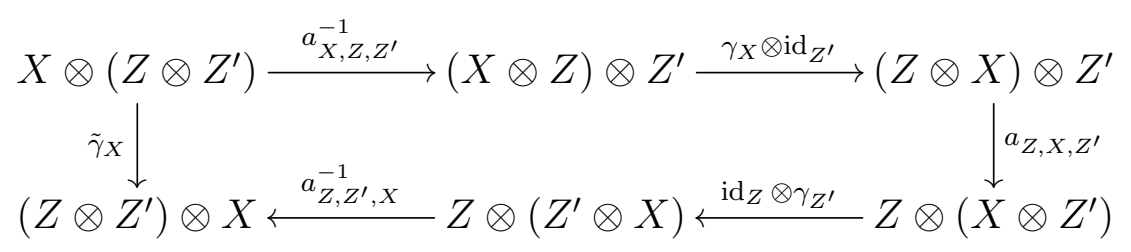

The associativity is the same as in $\mathcal{C}$ (a tedious calculation shows that $a_{X, Y, Z}$ is indeed a morphism in $\mathcal{Z}(\mathcal{C}))$ and the unit object is $\left(\mathbb{1}, r^{-1} l\right)$.

The category $\mathcal{Z}(\mathcal{C})$ is braided. The braiding is defined by

$$
c_{(Z, \gamma),\left(Z^{\prime}, \gamma^{\prime}\right)}=\gamma_{Z}^{\prime} \quad(Z, \gamma),\left(Z^{\prime}, \gamma^{\prime}\right) \in \mathcal{Z}\left(\operatorname{Vec}_{G}^{\omega}\right)
$$


For the remainder of this document, we fix a finite group $G$ with unit element $e$ and a normalized 3-cocycle, $\omega$. Also, we will use the shorthand ${ }^{f} g$ instead of $f g f^{-1}$, for any $f, g \in G$.

Remark 2.2.1. The Drinfeld center is the categorical version of a construction called Drinfeld double. Given a Hopf algebra $H$, its Drinfeld double $D(H)$ is another Hopf algebra whose category of representations is braided. In fact, $\operatorname{Rep}(D(H))$ is equivalent to $\mathcal{Z}(\operatorname{Rep}(H))$. For a detailed exposition on Hopf algebras and Drinfeld doubles see [16].

Definition 2.2.3. First, define

$$
\begin{aligned}
\omega\left(g, g^{\prime} ; h\right) & :=\frac{\omega\left(g, g^{g^{\prime}} h, g^{\prime}\right)}{\omega\left(g g^{\prime} h, g, g^{\prime}\right) \omega\left(g, g^{\prime}, h\right)}, \\
\omega(g ; f, h) & :=\omega(g, f, h) \omega\left({ }^{g} f, g, h\right)^{-1} \omega\left({ }^{g} f,{ }^{g} h, g\right),
\end{aligned}
$$

for $f, g, g^{\prime}, h \in G$. Using this, we define the braided category $\mathcal{Z}(G, \omega)$ as follows. Objects are $G$-graded vector spaces $V=\bigoplus_{g \in G} V_{g}$ with a linear map $\triangleright: \mathbb{C}^{\omega} G \otimes V \rightarrow V$ such that $1 \triangleright v=v$ for all $v \in V$,

$$
(g h) \triangleright v=\omega(g, h ; k)(g \triangleright(h \triangleright v)), \quad g, h, k \in G, \quad v \in V_{k},
$$

satisfying the following compatibility condition:

$$
g \triangleright V_{h} \subseteq V_{g h g^{-1}}, \quad g, h \in G .
$$

Objects in $\mathcal{Z}(G, \omega)$ are called Yetter-Drinfeld modules.

Morphisms in $\mathcal{Z}(G, \omega)$ are $G$-linear, $G$-homogeneous maps. The tensor product of $V=$ $\oplus_{g \in G} V_{g}$ and $W=\oplus_{g \in G} W_{g}$ is $V \otimes W$ as a vector space, with

$$
(V \otimes W)_{g}=\bigoplus_{h \in G} V_{h} \otimes W_{h^{-1} g}
$$

and for all $v \in V_{g}, w \in W_{l}$,

$$
h \triangleright(v \otimes w)=\omega(h ; g, l)(h \triangleright v) \otimes(h \triangleright w) .
$$

For $V, W, Z \in \mathcal{Z}(G, \omega)$ the associativity constrain is defined by

$$
\begin{aligned}
a_{V, W, Z}: & (V \otimes W) \otimes Z \\
\left(v_{g} \otimes w_{h}\right) \otimes z_{k} & \mapsto \omega(g, h, k) v_{g} \otimes\left(w_{h} \otimes z_{k}\right)
\end{aligned}
$$

for all $g, h, k \in G, v_{g} \in V_{g}, w_{h} \in W_{h}, z_{k} \in Z_{k}$. The unit object is denoted by $\mathbb{1}$ and it is a copy of $\mathbb{C}$ in the component of trivial degree, with the trivial representation of $G$. The category $\mathcal{Z}(G, \omega)$ is braided, with braiding $c_{V, W}: V \otimes W \rightarrow W \otimes V, V, W \in \mathcal{Z}(G, \omega)$ :

$$
c_{V, W}(v \otimes w)=(g \triangleright w) \otimes v, \quad g \in G, v \in V_{g}, w \in W .
$$




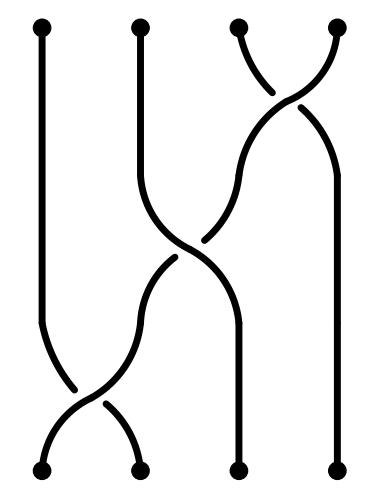

Figure 2.1: Example of an element in $\mathcal{B}_{4}$

Proposition 2.2.1. The categories $\mathcal{Z}\left(\operatorname{Vec}_{g}^{\omega}\right)$ and $\mathcal{Z}(G, \omega)$ are isomorphic as braided categories

Proof. See [6, Proposition 3.1].

From now on, we will refer to monoidal categories just by $\mathcal{C}$. The rest of the structure will be clear from context.

\section{Representations of the braid group}

Braided categories give rise to representations of a the Braid Group. As the name suggests, elements of the braid group are isotopy classes of braidings of $n$ strands. An example of an element, with $n=4$, is depicted in Figure 2.2. The operation in this group is composition of braids. A more formal definition will be given in the following, using generators and relations.

Definition 2.2.4. The braid group in $n$-strands, $\mathcal{B}_{n}$, is the group generated by $n-1$ elements $\left\{\sigma_{i}\right\}_{i=1, \ldots, n-1}$, subject to the relations

- $\sigma_{i} \sigma_{j}=\sigma_{j} \sigma_{i}$ for $|i-j|>1$.

- $\sigma_{i} \sigma_{i+1} \sigma_{i}=\sigma_{i+1} \sigma_{i} \sigma_{i+1}$ for $i=1, \ldots, n-2$.

Definition 2.2.5. Given a braided category $\mathcal{C}$ and an object $X$ of $\mathcal{C}$, we write $X^{\otimes n}$ for the object $(\ldots((X \otimes X) \otimes X) \ldots \otimes X) \otimes X$ ( $n$ copies $)$. For each generator of $\mathcal{B}_{n}, \sigma_{i}$, we define an automorphism $\sigma_{i}^{\prime} \in \operatorname{End}\left(X^{\otimes n}\right)$ as follows. We write $X_{i}$ for the $i$-th copy of $X$. Then

$$
\begin{aligned}
\sigma_{i}^{\prime}=\left(a_{X_{1} \otimes \cdots \otimes X_{i-1}, X_{i+1}, X_{i}}^{-1} \otimes\right. & \left.\operatorname{id}_{X_{i+2} \otimes \cdots \otimes X_{n}}\right) \circ \\
& \left(\operatorname{id}_{X_{1} \otimes \cdots X_{i-1}} \otimes c_{X_{i}, X_{i+1}} \otimes\right. \\
& \left.\operatorname{id}_{X_{i+2} \otimes \cdots \otimes X_{n}}\right) \circ \\
& \left(a_{X_{1} \otimes \cdots \otimes X_{i-1}, X_{i}, X_{i+1}} \otimes \operatorname{id}_{X_{i+2} \otimes \cdots \otimes X_{n}}\right)
\end{aligned}
$$


Proposition 2.2.2. A linear representation $\psi_{X}: \mathcal{B}_{n} \rightarrow \operatorname{End}_{\mathbb{C}}\left(\operatorname{Hom}_{\mathcal{C}}\left(\mathbb{1}, X^{\otimes n}\right)\right)$ is defined by assigning to each generator $\sigma_{i}$ the linear transformation

$$
\begin{aligned}
\operatorname{Hom}_{\mathcal{C}}\left(\mathbb{1}, X^{\otimes n}\right) & \rightarrow \operatorname{Hom}_{\mathcal{C}}\left(\mathbb{1}, X^{\otimes n}\right) \\
f & \mapsto \sigma_{i}^{\prime} \circ f
\end{aligned}
$$

Proof. See [16].

\subsection{Lagrangian Algebras}

Lagrangian algebras are a family of objects in $\mathcal{Z}(G, \omega)$. They came to the attention of researchers first in the physics literature, though it took some time before it was realized that they were indeed the right object to consider. Their formal definition is the following:

Definition 2.3.1. Following [6, Corollary 3.17], we will describe the Lagrangian algebra on $\mathcal{Z}(G, \omega)$ associated to a pair $(H, \gamma)$, where $H \subseteq G$, is a subgroup and $\gamma: H \times H \rightarrow \mathbb{C}^{\times}$a map such

$$
\frac{\gamma(a b, c) \gamma(a, b)}{\gamma(a, b c) \gamma(b, c)}=\omega(a, b, c), \quad a, b, c \in H .
$$

Let $\mathbb{C}_{\gamma}[H]=\oplus_{h \in H} \mathbb{C} u_{h}$ the group algebra of $H$. It is an object in $\mathcal{Z}\left(H,\left.\omega\right|_{H}\right)$, where the $H$-action is given by

$$
h_{1} \triangleright u_{h_{2}}=\epsilon\left(h_{1}, h_{2}\right) u_{h_{1} h_{2} h_{1}^{-1},} \quad \epsilon\left(h_{1}, h_{2}\right):=\frac{\gamma\left(h_{1}, h_{2}\right)}{\gamma\left({ }^{h_{1}} h_{2}, h_{1}\right)}, \quad h_{1}, h_{2} \in H,
$$

and grading $\left|u_{h}\right|=h$ for all $h \in H$.

Let $\operatorname{Map}\left(G, \mathbb{C}_{\gamma}[H]\right)$ be the vector space of all set-theoretic maps from $G$ to $\mathbb{C}_{\gamma}[H]$. With the grading given by

$$
|a|=f \quad \Leftrightarrow \quad \forall x \in G \quad|a(x)|=x^{-1} f x
$$

and twisted $G$-action

$$
(g \triangleright a)(x):=\omega\left(x^{-1}, g^{-1} ;|a|\right)^{-1} a\left(g^{-1} \triangleright x\right), \quad g, x \in G .
$$

we can see $\operatorname{Map}\left(G, \mathbb{C}_{\gamma}[H]\right)$ as an object in $\mathcal{Z}(G, \omega)$. The Lagrangian algebra $L(H, \gamma)$ is the subspace of $\operatorname{Map}\left(G, \mathbb{C}_{\gamma}[H]\right)$

$$
L(H, \gamma):=\left\{a \in \operatorname{Maps}\left(G, \mathbb{C}_{\gamma}[H]\right) \mid a(x h)=\omega\left(h^{-1}, x^{-1} ;|a|\right) h^{-1} \triangleright a(g)\right\},
$$

see [6] for more details. 
In the rest of this section we will try to motivate this definition. We will not get into any details, however. We will use the notion of (indecomposable) module category defined in [11, Definition 7.1.2], as well as those of an algebra in a monoidal category [11, Definition 7.8.1] and modules over such algebras [11, Definition 7.8.5].

Proposition 2.3.1. There is a bijection between indecomposable semisimple module categories over $\operatorname{Vec}_{G}^{\omega}$ and pairs $(H, \gamma)$ where $H$ is a subgroup of $G$ and $\gamma \in C^{2}\left(H, \mathbb{C}^{\times}\right)$such that $d^{3} \gamma=\left.\omega\right|_{H}$, defined up to conjugation.

Proof. Given such a module category, $G$ acts transitively on its simples. Thus they are in bijection with a collection of cosets $G / H$, for some subgroup $H$ defined up to conjugation. The associativity constraints of the module category, restricted to $H$, give the 2-cocycle.

Fix $H$ and $\gamma$ as in Proposition 2.3.1. The vector space $\mathbb{C}_{\gamma}[H]$ that was part of Definition 2.3.1 has a structure of algebra. Its multiplication is given by

$$
u_{g} \cdot u_{h}=\gamma(g, h) u_{g h}
$$

If we write $M_{H, \gamma}$ for the module category given by Proposition 2.3 .1 and $\left(\operatorname{Vec}_{G}^{\omega}\right)_{\mathbb{C}_{\gamma}[H]}$ for the category of modules of $\mathbb{C}_{\gamma}[H]$ in $\operatorname{Vec}_{G}^{\omega}$, we get the following:

Proposition 2.3.2. The module category $M_{H, \gamma}$ is equivalent to $\left(\operatorname{Vec}_{G}^{\omega}\right)_{\mathbb{C}_{\gamma}[H]}$.

Proof. This is an example of a more general fact. Indeed, any module category $\mathcal{M}$ over $\operatorname{Vec}_{G}^{\omega}$ is equivalent to the category of modules over the internal Hom from $\mathcal{M}$ to itself. See [11, Theorem 7.10.1].

Now define $\left(M_{H, \gamma}\right)_{\operatorname{Vec}_{G}^{\omega}}^{*}$ to be the category of module functors, see [11, Definition 7.2.1]. This is a module category over $\mathcal{Z}\left(\operatorname{Vec}_{G}^{\omega}\right)$. Thus by the same token as in Proposition 2.3.2, there exists an algebra $A$ in $\mathcal{Z}\left(\operatorname{Vec}_{G}^{\omega}\right)$ such that $\mathcal{Z}\left(\operatorname{Vec}_{G}^{\omega}\right)_{A} \cong\left(M_{H, \gamma}\right)_{\operatorname{Vec}_{G}^{\omega}}^{*}$. The algebra $A$ is precisely $L[H, \gamma]$. 


\section{Chapter 3}

\section{Monomiality of $\psi_{L[H, \gamma]}$}

Consider the representation $\psi_{X}$ from Definition 2.2.5, with $\mathcal{C}=\mathcal{Z}(G, \omega)$ and $X=L[H, \gamma]$. This representation is interesting to physicists. Roughly speaking, $\operatorname{Hom}_{\mathcal{Z}(G, \omega)}\left(\mathbb{1}, L[H, \gamma]^{\otimes n}\right)$ is the ground state of a quantum mechanical system. Moreover, it is the computational space for a model of topological quantum computation. This representation encodes the quantum gates of the model, so it is of interest to determine precisely the image of the representation. More details can be found in [5]. The goal of this chapter is to study this representation.

\subsection{Monomial representations}

In this section we recall some basic definitions and results on monomial representations of groups.

Definition 3.1.1. A monomial space is a triple $\mathbf{V}=\left(V, X,\left(V_{x}\right)_{x \in X}\right)$ where,

(i) $\mathrm{V}$ is a finite dimensional complex vector space.

(ii) $\mathrm{X}$ is a finite set.

(iii) $\left(V_{x}\right)_{x \in X}$ is a family of one dimensional subspaces of $V$, indexed by $X$, such that $V=$ $\bigoplus_{x \in X} V_{x}$

Let $G$ be a group. By a monomial representation of $G$ on $\mathbf{V}$ we mean a group homomorphism

$$
\Gamma: G \rightarrow \mathrm{GL}(V)
$$

such that for every $g \in G, \Gamma(g)$ permutes the $V_{x}$ 's; hence, $\Gamma$ induces an action by permutation of $G$ on $X$. We will denote $\Gamma(g)(v)$ just by $g \triangleright v$.

If $V$ is a representation of $G$, we denote by $V^{G}$ the subspace of $G$-invariant vectors, i.e.,

$$
V^{G}=\{v \in V: g \triangleright v=v, \text { for all } g \in G\}
$$


For each $x \in X$, we will denote $\operatorname{Stab}_{G}(x)$ the stabilizer of $x$ and by $\mathcal{O}_{G}(x)$ the $G$-orbit of $x$. For $G$ finite, and a representation $V$ define

$$
\operatorname{Av}_{G}: V \rightarrow V, \quad v \mapsto \frac{1}{|G|} \sum_{g \in G} g \triangleright v .
$$

It is easy to see that $\operatorname{Av}_{G}$ is a $G$-linear projection onto $V^{G}$. We define,

$$
\operatorname{Av}_{G}\left(V_{\mathcal{O}}\right):=\operatorname{Av}_{G}\left(V_{x}\right), \quad x \in \mathcal{O}(x),
$$

since for any $x^{\prime} \in \mathcal{O}_{G}(x), \operatorname{Av}_{G}\left(V_{x}\right)=\operatorname{Av}_{G}\left(V_{x^{\prime}}\right)$.

We say that an element $x \in X$ is regular under the monomial action of $G$ if $\Gamma(g)$ is the identity map on $V_{x}$, for all $g \in \operatorname{Stab}_{G}(x)$.

Let us write $X / G$ for the set of orbits of the action of $G$ on $X$ and $\tilde{X}$ for the regular ones.

Proposition 3.1.1. [15, Lemma 9.1] Let $\mathbf{V}=\left(V, X,\left(V_{x}\right)_{x \in X}\right)$ be a monomial representation of $G$.

(a) $x \in X$ is a regular element if and only if $\operatorname{Av}_{G}\left(V_{x}\right) \neq 0$.

(b) If $x \in X$ is a regular element under the monomial action of $G$, then so are all elements in the $G$-orbit of $x$.

(c) The triple

$$
\mathbf{V}^{G}=\left(V^{G}, \tilde{X},\left(\operatorname{Av}_{G}\left(V_{\mathcal{O}}\right)\right)_{\mathcal{O} \in \tilde{X}}\right)
$$

is a monomial space.

(d) The dimension of $V^{G}$ is equal to the number of regular G-orbits under the monomial action of $G$ on $X$.

Let $\mathbf{V}=\left(V, X,\left(V_{x}\right)_{x \in X}\right)$ and $\mathbf{V}^{\prime}=\left(V^{\prime}, Y,\left(V_{y}^{\prime}\right)_{y \in Y}\right)$ be monomial spaces. A linear isomorphism $T: V \rightarrow V^{\prime}$ is called an isomorphism of monomial spaces if $T\left(V_{x}\right)=V_{y}^{\prime}$ for any $x \in X$.

Proposition 3.1.2. Let $\mathbf{V}=\left(V, X,\left(V_{x}\right)_{x \in X}\right)$ and $\mathbf{V}^{\prime}=\left(V^{\prime}, Y,\left(V_{y}^{\prime}\right)_{y \in Y}\right)$ be monomial representations of a finite group $G$. If $T: V \rightarrow V^{\prime}$ is a $G$-linear isomorphism of monomial spaces, then $\left.T\right|_{V^{G}}: \mathbf{V}^{G} \rightarrow \mathbf{V}^{\prime G}$ is an isomorphism of monomial spaces.

Proof. Clearly, $\left.T\right|_{V^{G}}: V^{G} \rightarrow V^{\prime G}$ is a linear isomorphism. Let $x \in X$ be a regular element. Since $T$ is an isomorphism of monomial spaces, there is some $y \in Y$ such that $T\left(V_{x}\right)=V_{y}^{\prime}$. In that case:

$$
\operatorname{Av}_{G}\left(V_{y}^{\prime}\right)=\operatorname{Av}_{G}\left(T\left(V_{x}\right)\right)=T\left(\operatorname{Av}_{G}\left(V_{x}\right)\right)
$$


This implies $y$ is regular, because $\operatorname{Av}_{G}\left(V_{x}\right) \neq\{0\}$ and $T$ is an isomorphism. It also says $\left.T\right|_{V^{G}}\left(\operatorname{Av}_{G}\left(V_{\mathcal{O}(x)}\right)\right)=\operatorname{Av}_{G}\left(V_{\mathcal{O}(y)}^{\prime}\right)$, which means $\left.T\right|_{V^{G}}$ is an isomorphism of monomial spaces.

\subsection{Monomial representation of the braid group}

In this section we introduce the notion of monomial twisted Yetter-Drinfeld and prove that the representation of the braid groups $\mathcal{B}_{n}$ over $\operatorname{Hom}_{\mathcal{Z}(G, \omega)}\left(\mathbb{C}, V^{\otimes n}\right)$ is monomial if $V$ is monomial.

We begin with the notion of a crossed $G$-set. This was first introduced by Whitehead in the context of algebraic topology [22].

\section{Crossed $G$-sets}

Let $G$ be a group. A (left) crossed $G$-set is a left $G$-set $X$ and a grading function $|-|: X \rightarrow G$ such that

$$
|g x|=g|x| g^{-1}
$$

for all $x \in X, g \in G$. If $X$ and $Y$ are crossed $G$-sets, a $G$-equivariant map $f: X \rightarrow Y$ is a morphism of crossed $G$-sets if $|f(x)|=|x|$ for all $x \in X$.

If $X$ and $Y$ are crossed $G$-sets, the cartesian product $X \times Y$ is a crossed $G$-set with the diagonal action and grading map $|(x, y)|=|x||y|$.

The category of crossed $G$-sets is a braided category with braiding

$$
\begin{aligned}
c_{X, Y}: X \times Y & \rightarrow Y \times X \\
(x, y) & \mapsto(|x| \triangleright y, x) .
\end{aligned}
$$

Thus, given a crossed $G$-set $X$ the braid group $\mathcal{B}_{n}$ acts on $X^{n}$, in the following way

$$
\sigma_{i}^{\prime}:=\operatorname{id}_{X^{i-1}} \times c_{X, X} \times \operatorname{id}_{X^{n-(i-1)}} .
$$

\section{Monomial objects of $\mathcal{Z}(G, \omega)$}

Definition 3.2.1. A monomial Yetter-Drinfeld module is a monomial space $\mathbf{V}=\left(V, X,\left(V_{x}\right)_{x \in X}\right)$ such that $V \in \mathcal{Z}(G, \omega)$, the twisted $G$-action $\triangleright$ permutes the $V_{x}$ 's and each $V_{x}$ is $G$ homogeneous.

Remark 3.2.1. (a) If $\mathbf{V}=\left(V, X,\left(V_{x}\right)_{x \in X}\right)$ is a monomial Yetter-Drinfeld module, the set $X$ is a crossed $G$-set with the induced $G$-action and the grading map.

(b) If $\mathbf{V}=\left(V, X,\left(V_{x}\right)_{x \in X}\right)$ is a monomial Yetter-Drinfeld module, the action of $G$ on $\left(V_{e}, X_{e},\left(V_{x}\right)_{x \in X_{e}}\right)$ is monomial, where $X_{e}:=\{x \in X:|x|=e\}$ and $V_{e}=\oplus_{x \in X_{e}} V_{x}$. 
Theorem 3.2.1. If $\mathbf{V}=\left(V, X,\left(V_{x}\right)_{x \in X}\right)$ is a monomial Yetter-Drinfeld module in $\mathcal{Z}(G, \omega)$, then

(a) the action of $\mathcal{B}_{n}$ on $\operatorname{Hom}_{\mathcal{Z}(G, \omega)}\left(\mathbb{C}, V^{\otimes n}\right)$ is monomial,

(b) the dimension of $\operatorname{Hom}_{\mathcal{Z}(G, \omega)}\left(\mathbb{C}, V^{\otimes n}\right)$ is equal to the number of regular $G$-orbits under the monomial action of $G$ on

$$
\left(X^{n}\right)_{e}:=\left\{\left(x_{1}, \ldots, x_{n}\right):\left|x_{1}\right| \cdots\left|x_{n}\right|=e\right\} .
$$

Proof. The action of $G$ on $\left(V_{e}^{\otimes n},\left(X^{n}\right)_{e},\left(V_{x}\right)_{x \in X_{e}}\right)$ is monomial. Hence by Proposition 3.1.1, the triple

$$
\mathbf{V}_{e}^{G}:=\left(\left(\left(V_{e}^{\otimes n}\right)^{G}, \widetilde{\left(X^{n}\right)_{e}},\left(\operatorname{Av}_{G}\left(\left(V_{e}^{\otimes n}\right)_{\mathcal{O}}\right)\right)_{\mathcal{O} \in\left(\widetilde{\left.X^{n}\right)_{e}}\right.}\right)\right.
$$

is a monomial space. Since $\operatorname{Hom}_{\mathcal{Z}(G, \omega)}\left(\mathbb{C}, V^{\otimes n}\right)=\left(V^{\otimes n}\right)_{e}^{G}$, and each of the automorphisms $\sigma^{\prime}$ is a morphism in $\mathcal{Z}(G, \omega)$, hence $\left.\sigma^{\prime}\right|_{V_{e}^{\otimes n}}:\left(V_{e}^{\otimes n},\left(X^{n}\right)_{e},\left(V_{x}\right)_{x \in X_{e}}\right) \rightarrow\left(V_{e}^{\otimes n},\left(X^{n}\right)_{e},\left(V_{x}\right)_{x \in X_{e}}\right)$ is a $G$-linear isomorphism of monomial spaces. It follows from Proposition 3.1.2 that $\left.\sigma^{\prime}\right|_{\left(V_{e}^{\otimes n}\right)^{G}}$ is an isomorphism of monomial spaces. Thus, the linear representation

$$
\begin{aligned}
\rho_{n}: \mathcal{B}_{n} & \rightarrow \mathrm{GL}\left(\left(V_{e}^{\otimes n}\right)^{G}\right) \\
\sigma & \mapsto \sigma^{\prime},
\end{aligned}
$$

is a monomial representation of $\mathcal{B}_{n}$. The second part follows immediately from Proposition 3.1.1.

Remark 3.2.2. Similar ideas to those in the proof of Theorem 3.2.1 can be used to show that for any object $X \in \mathcal{Z}(G, \omega)$, the image of the representation of $\mathcal{B}_{n}$ on $\operatorname{End}_{\mathcal{Z}(G, \omega)}\left(X^{\otimes n}\right)$ is finite. This was shown in [10]. The main idea there is that $\mathcal{Z}\left(\operatorname{Vec}_{G}^{\omega}\right)$ is equivalent to the category of modules over the Drinfeld double of $G, \operatorname{Rep}(\mathcal{Z}(G, \omega))$. There, every object is a quotient of a free module, hence it suffices to show finiteness on the regular module. This is done by a direct calculation similar to the ones we do here. We should remark that while that result is more general, we need the specific basis and matrices of the representations $\psi_{L[H, \gamma]}$ for specific applications in Chapter 4.

\section{Monomial matrices of the braid representation}

In this subsection we obtain concrete formulas for the monomial braid representations associated to a monomial Yetter-Drinfeld module.

Let $\mathbf{V}=\left(V, X,\left(V_{x}\right)_{x \in X}\right)$ be a monomial Yetter-Drinfeld module. If we fix non-zero vectors $\mathcal{S}:=\left\{v_{x} \in V_{x}: x \in X\right\}$, the twisted $G$-action defines a map

$$
\lambda_{X}: G \times X \rightarrow \mathbb{C}^{\times},
$$

by $g \triangleright v_{x}=\lambda_{X}(g ; x) v_{g x}$, where $g \in G, x \in X$. 
For the monomial Yetter-Drinfeld module $\mathbf{V}^{\otimes n}=\left(V^{\otimes n}, X^{n},\left(V_{x}\right)_{x \in X^{n}}\right)$ and the basis $\mathcal{S}^{\otimes n}:=\left\{v_{x_{1}} \otimes \cdots \otimes v_{x_{n}}: x_{i} \in X, 1 \leq i \leq n\right\}$, the action is determined by the map $\lambda_{X^{n}}: G \times X^{n} \rightarrow \mathbb{C}^{\times}$,

$$
\begin{aligned}
& \lambda_{X^{n}}\left(g ; x_{1}, \ldots, x_{n}\right):=\prod_{i=1}^{n} \lambda_{X}\left(g ; x_{i}\right) \omega\left(g ;\left|x_{1}\right|\left|x_{2}\right| \cdots\left|x_{n-1}\right|,\left|x_{n}\right|\right) \times \\
& \omega\left(g ;\left|x_{1}\right| \cdots\left|x_{n-2}\right|,\left|x_{n-1}\right|\right) \cdots \omega\left(g ;\left|x_{1}\right|,\left|x_{2}\right|\right),
\end{aligned}
$$

that is,

$$
g \triangleright\left(v_{x_{1}} \otimes \cdots \otimes v_{x_{n}}\right)=\lambda_{X^{n}}\left(g ; x_{1}, \ldots, x_{n}\right)\left(v_{g x_{1}} \otimes \cdots \otimes v_{g x_{n}}\right),
$$

for all $g \in G, x_{1}, x_{2} \ldots, x_{n} \in X$. Hence an element $\left(x_{1}, \ldots, x_{n}\right) \in\left(X^{n}\right)_{e}$ is regular if and only if

$$
\lambda_{X^{n}}\left(g ; x_{1} \ldots, x_{n}\right)=1, \quad \text { for all } g \in \bigcap_{i=1}^{n} \operatorname{Stab}\left(x_{i}\right) .
$$

Let $\mathcal{R} \subset X_{e}^{n}$ be a set of representatives of the regular orbits of $X_{e}^{n}$. Let $\mathcal{S}_{\text {reg }}=\left\{v_{x_{1}} \otimes\right.$ $\left.\cdots \otimes v_{x_{n}}:\left(x_{1}, \ldots, x_{n}\right) \in R\right\}$. By Proposition (3.1.1) the set $\left\{\operatorname{Av}_{G}(v): v \in \mathcal{S}_{\text {reg }}\right\}$ is a basis of $\left(V^{\otimes n}\right)_{e}^{G}$.

In order to express the action of the generator $\sigma_{i} \in \mathcal{B}_{n}$ in terms of $\left\{\operatorname{Av}_{G}(v): v \in\right.$ $\left.\mathcal{S}_{\text {reg }}\right\}$, for each $\mathbf{x}=\left(x_{1}, \ldots, x_{n}\right) \in \mathcal{R}$ choose $g_{\mathbf{x}} \in G$ such that $g_{\mathbf{x}} \triangleright \sigma_{i}^{\prime}(\mathbf{x})=\mathbf{y}$, where $\mathbf{y} \in \mathcal{R}$ and $\sigma_{i}^{\prime}(\mathbf{x})=\left(x_{1}, \cdots, x_{i-1},\left|x_{i}\right| \triangleright x_{i+1}, x_{i}, \cdots, x_{n}\right)$. Hence there is $\beta_{i, \mathbf{x}} \in \mathbb{C}^{\times}$such that $g_{\mathbf{x}} \triangleright \sigma_{i}^{\prime}\left(v_{x_{1}} \otimes \cdots v_{x_{n}}\right)=\beta_{i, \mathbf{x}} v_{y_{1}} \otimes \cdots \otimes v_{y_{n}}$.

Since the action of the generator $\sigma_{i} \in \mathcal{B}_{n}$ is given by

$$
\begin{gathered}
\sigma^{\prime}\left(v_{x_{1}} \otimes \cdots v_{x_{n}}\right)=\omega^{-1}\left(\left|x_{1}\right| \cdots\left|x_{i-1}\right|,\left|x_{i+1}\right|,\left|x_{i}\right|\right) \times \\
\lambda_{X}\left(\left|x_{i}\right| ; x_{i+1}\right) \omega\left(\left|x_{1}\right| \cdots\left|x_{i-1}\right|,\left|x_{i}\right|,\left|x_{i+1}\right|\right) \times \\
v_{x_{1}} \otimes \cdots \otimes v_{x_{i-1}} \otimes v_{\left|x_{i}\right| x_{i+1}} \otimes v_{x_{i}} \otimes \cdots \otimes v_{x_{n}} .
\end{gathered}
$$

we have that

$$
\begin{aligned}
& \beta_{i, \mathbf{x}}=\omega^{-1}\left(\left|x_{1}\right| \cdots\left|x_{i-1}\right|,\left|x_{i+1}\right|,\left|x_{i}\right|\right) \times \\
& \lambda_{X}\left(\left|x_{i}\right| ; x_{i+1}\right) \omega\left(\left|x_{1}\right| \cdots\left|x_{i-1}\right|,\left|x_{i}\right|,\left|x_{i+1}\right|\right) \lambda_{X^{n}}\left(g_{\mathbf{x}} ; \sigma_{i}^{\prime}(\mathbf{x})\right) .
\end{aligned}
$$

Theorem 3.2.2. Let $G$ be a finite group, $\omega \in Z^{3}\left(G, \mathbb{C}^{\times}\right)$and $\mathbf{V}=\left(V, X,\left(V_{x}\right)_{x \in X}\right)$ be a monomial Yetter-Drinfeld module. Let $Y$ be the set of all regular elements in $X_{e}^{n}$ and $\mathcal{R} \subset Y$ a set of representatives of the G-orbits of $Y$.

(a) The projection $\pi: Y \rightarrow \mathcal{R}$ is map of $\mathcal{B}_{n}$-sets. The image of $\mathbf{x} \in \mathcal{R}$ by the generator $\sigma_{i} \in \mathcal{B}_{n}$ will be denoted by $\sigma_{i} \triangleright \mathbf{x}$. 
(b) Let $\mathcal{S}_{\text {reg }}=\left\{v_{x_{1}} \otimes \cdots \otimes v_{x_{n}}:\left(x_{1}, \ldots, x_{n}\right) \in \mathcal{R}\right\}$. The action of the generator $\sigma_{i} \in \mathcal{B}_{n}$ in the basis $\left\{\operatorname{Av}_{G}\left(v_{\mathbf{x}}\right): \mathbf{x} \in \mathcal{R}\right\}$ is given by

$$
\sigma_{i}\left(\operatorname{Av}_{G}\left(v_{\mathbf{x}}\right)\right)=\beta_{i, \mathbf{x}} \operatorname{Av}_{G}\left(v_{\sigma_{i} \triangleright \mathbf{x}}\right),
$$

where $\beta_{i, \mathbf{x}}$ was defined in (3.4).

Proof. The first part is consequence of Theorem 3.2.1.

For the second part, recall that the number $\beta_{i, \mathbf{x}}$ and the element $g_{\mathbf{x}} \in G$ are such that

$$
g_{\mathbf{x}} \triangleright \sigma\left(v_{\mathbf{x}}\right)=\beta_{i, \mathbf{x}} v_{\sigma_{i} \triangleright \mathbf{x}} .
$$

Hence,

$$
\begin{aligned}
\sigma_{i}\left(\operatorname{Av}_{G}\left(v_{\mathbf{x}}\right)\right) & =\operatorname{Av}_{G}\left(\sigma_{i}\left(v_{\mathbf{x}}\right)\right) \\
& =g_{\mathbf{x}} \triangleright \operatorname{Av}_{G}\left(\sigma_{i}\left(v_{\mathbf{x}}\right)\right) \\
& =\operatorname{Av}_{G}\left(g_{\mathbf{x}} \triangleright \sigma_{i}\left(v_{\mathbf{x}}\right)\right) \\
& =\operatorname{Av}_{G}\left(\beta_{i, \mathbf{x}} v_{\sigma_{i} \triangleright \mathbf{x}}\right) \\
& =\beta_{i, \mathbf{x}} \operatorname{Av}_{G}\left(v_{\sigma_{i} \triangleright \mathbf{x}}\right) .
\end{aligned}
$$

Example 3.2.1. Let $G$ be a finite group and $X$ be a left crossed $G$-set. Then the linearization $V_{X}:=\oplus_{x \in X} \mathbb{C} x$ is a (untwisted) Yetter-Drinfeld module in $\mathcal{Z}\left(\operatorname{Vec}_{G}\right)$. Clearly $\lambda_{X} \equiv 1$, thus every element in $\left(X^{n}\right)_{e}$ is regular. Hence the canonical projection

$$
\left(X^{n}\right)_{e} \rightarrow\left(X^{n}\right)_{e} / / G,
$$

is an epimorphism of $\mathcal{B}_{n}$-sets. In other words, the linear representation of $\mathcal{B}_{n}$ on $\operatorname{Hom}_{\mathcal{Z}(G, \omega)}\left(\mathbb{C}, V_{X}^{\otimes n}\right)$ is the linearization of the permutation action of $\mathcal{B}_{n}$ on $\left(X^{n}\right)_{e} / / G$.

\subsection{Braid groups representations associated to Lagrangian algebras}

In this section we prove that every Lagrangian algebra $\mathcal{Z}(G, \omega)$ has a canonical monomial structure. Then the results of Section 3.2 can be applied to Lagrangian algebras in $\mathcal{Z}(G, \omega)$.

\section{Monomial structure of the Lagrangian algebras $L(H, \gamma)$}

Let $G$ be a group and $H \subset G$ a subgroup. We can regard $G \times H$ as a left $H$-set with actions given $h \triangleright\left(g, h^{\prime}\right)=\left(g h^{-1}, h h^{\prime} h^{-1}\right)$. Then we can consider the set of $H$-orbits that we will denote by $G \times_{H} H$. The set $G \times_{H} H$ is equipped with a left $G$-action given by left multiplication on the first component. 
Definition 3.3.1. Let $L(H, \gamma)$ be a Lagrangian. For each $g \in G, f \in H$, define $\chi_{g, f} \in$ $L(H, \gamma)$ by

$$
\chi_{g, f}(x)= \begin{cases}0, & x \notin g H \\ \omega\left(h^{-1}, g^{-1} ;{ }^{g} f\right) \epsilon\left(h^{-1}, f\right) e_{h f h^{-1}}, & x=g h, \text { where } h \in H .\end{cases}
$$

Remark 3.3.1. The function $\chi_{g, h}$ can be characterized as the unique map in $L(H, \gamma)$ with support $g H$ and such that $\chi_{g, h}(g)=e_{h}$.

Lemma 3.3.1. Let $L(H, \gamma)$ be a Lagrangian algebra in $\mathcal{Z}(G, \omega)$. Then

$$
\begin{array}{cc}
\chi_{g h, f}=\omega\left(h,(g h)^{-1} ;{ }^{g h} f\right) \epsilon(h, f) \chi_{g^{,} f}, & g \in G, f, h \in H . \\
l \triangleright \chi_{g, f}=\omega\left((l g)^{-1}, l^{-1} ;{ }^{g} f\right) \chi_{l g, f}, & g, l \in G, h \in H .
\end{array}
$$

Proof. (3.5). Since the supports of $\chi_{g h, f}$ and $\chi_{g,{ }^{h} f}$ are $g H$, and

$$
\begin{aligned}
\chi_{g h, f}(g) & =\chi_{g h, f}\left(g h h^{-1}\right) \\
& =\omega\left(h,(g h)^{-1} ;{ }^{g h} f\right) \epsilon(h, f) \chi_{g,{ }^{h} f}(g),
\end{aligned}
$$

we obtain (3.5).

(3.6). By the definition of the action of $G$ we have

$$
\begin{aligned}
l \triangleright \chi_{g, f}(l g) & =\omega\left((l g)^{-1}, l^{-1} ;{ }^{g} f\right) \chi_{g, f}(g) \\
& =\omega\left((l g)^{-1}, l^{-1} ;{ }^{g} f\right) e_{f} .
\end{aligned}
$$

Since $l \triangleright \chi_{g, f}$ and $\chi_{g l, f}$ are supported in $g l H$, we get (3.6).

It follows from Lemma 3.3.1 that $\mathbb{C} \chi_{g h,{ }_{f} f}=\mathbb{C} \chi_{g, f}$. Then for any $(g, h) \in G \times_{H} H$ the space $\mathbb{C} \chi_{g, f}$ is well defined.

Theorem 3.3.1. Let $L(H, \gamma)$ be a Lagrangian algebra in $\mathcal{Z}(G, \omega)$. Then $L(H, \gamma)$ with the decomposition

$$
L(H, \gamma)=\bigoplus_{(g, h) \in G \times_{H} H} \mathbb{C} \chi_{g, h}
$$

is a monomial twisted Yetter-Drinfeld module.

Proof. First we will check that in fact the $\operatorname{sum} \sum_{(g, h) \in G \times{ }_{H} H} \mathbb{C} \chi_{g, h}$, is direct. Since $\operatorname{supp}\left(\chi_{g, f}\right)=$ $g H$, we have that $\chi_{g, f}$ and $\chi_{g^{\prime}, f}$ are linearly independent if $g H \neq g^{\prime} H$. Hence it is suffices to check linear independence of the collections $\left\{\chi_{g, f}\right\}_{f \in H}$, with $g$ fixed. But if $f \neq f^{\prime}$, $\left|\chi_{r, f}\right| \neq\left|\chi_{r, f^{\prime}}\right|$. It follows that the sum $\sum_{(g, h) \in G \times_{H} H} \mathbb{C} \chi_{g, h}$ is direct. 
In order to see that $L(H, \gamma)=\sum_{(g, h) \in G \times{ }_{H} H} \mathbb{C} \chi_{g, h}$, fix $\mathcal{R} \subset G$ a set of representative of the left coset of $H$ in $G$. Let $a \in L(H, \gamma)$. For each $r \in \mathcal{R}$, suppose

$$
a(r)=\sum_{f \in H} \lambda_{r, f} e_{f}
$$

Then we have

$$
a=\sum_{r \in \mathcal{R}, f \in H} \lambda_{r, f} \chi_{r, f} \in \sum_{(g, h) \in G \times_{H} H} \mathbb{C} \chi_{g, h}
$$

By (3.6) and the fact that $\left|\chi_{g, f}\right|=g f g^{-1}$, we obtain that $L(H, \gamma)$ is a monomial twisted Yetter-Drinfeld module.

Corollary 3.3.1. If $L(H, \gamma)$ is a Lagrangian algebra in $\mathcal{Z}(G, \omega)$, then

(a) the action of $\mathcal{B}_{n}$ on $\operatorname{Hom}_{\mathcal{Z}(G, \omega)}\left(\mathbb{C}, L(H, \gamma)^{\otimes n}\right)$ is monomial,

(b) the dimension of $\operatorname{Hom}_{\mathcal{Z}\left(\operatorname{Vec}_{G}^{\omega}\right)}\left(\mathbb{C}, L(H, \gamma)^{\otimes n}\right)$ is equal to the number of regular $G$-orbits under the monomial action of $G$ on

$$
\left(G \times_{H} H\right)_{e}^{n}:=\left\{\left(\left(g_{1}, h_{1}\right), \ldots,\left(g_{n}, h_{n}\right)\right): g_{1} h_{1} g_{1}^{-1} g_{2} h_{2} g_{2}^{-1} \cdots g_{n} h_{n} g_{n}^{-1}=e\right\}
$$

Proof. Follows from Theorem 3.3.1 and Theorem 3.2.1.

We will fix a set of representatives of the left cosets of $G$ in $H, \mathcal{R} \subset G$. Thus every element $g \in G$ has a unique factorization $g=r h, h \in H, r \in \mathcal{R}$. We assume $e \in \mathcal{R}$. The uniqueness of the factorization $G=\mathcal{R} H$ implies that there are well defined maps

$$
\triangleright: G \times \mathcal{R} \rightarrow \mathcal{R}, \quad \kappa: G \times \mathcal{R} \rightarrow H,
$$

determined by the condition

$$
g r=(g \triangleright r) \kappa(g, h), \quad g \in G, r \in \mathcal{R} .
$$

As crossed $G$-set we can identify $G \times_{H} H$ with $\mathcal{R} \times H$ with action

$$
g \triangleright(r, h):=\left(g \triangleright r,{ }^{\kappa(g, r)} h\right), \quad r \in \mathcal{R}, h \in H, g \in G,
$$

and grading map

$$
\begin{aligned}
|-|: \mathcal{R} \times H & \rightarrow G \\
(r, h) & \mapsto r h r^{-1} .
\end{aligned}
$$

It follows from Theorem (3.3.1) that $B_{\mathcal{R}}:=\left\{\chi_{r, h} \mid r \in \mathcal{R}, h \in H\right\}$ is a basis for $L(H, \gamma)$.

In order to apply the results of Subsection 3.2, we only need to compute the map $\lambda_{\mathcal{R} \times H}$ : $G \times(\mathcal{R} \times H) \rightarrow \mathbb{C}^{\times}$, such that 


$$
g \triangleright \chi_{r, h}=\lambda_{\mathcal{R} \times H}(g ; r, h) \chi_{g \triangleright r,{ }^{\kappa(g, r)} h}, \quad g \in G, r \in \mathcal{R}, h \in H .
$$

Using Lemma 3.3.1 we obtain that

$$
\lambda_{\mathcal{R} \times H}(g ; r, h)=\omega\left((g r)^{-1}, g^{-1} ;{ }^{r} h\right) \omega\left(\kappa(g, r),(g r)^{-1} ;{ }^{g r} h\right) \epsilon(\kappa(g, r), h),
$$

for all $g \in G, r \in \mathcal{R}, h \in H$.

By (3.2), we have that an element $t=\left(\left(r_{1}, h_{1}\right), \ldots,\left(r_{n}, h_{n}\right)\right) \in(\mathcal{R} \times H)_{e}^{n}$ is regular if and only if

$$
\lambda_{(\mathcal{R} \times H)^{n}}\left(g ;\left(r_{1}, h_{1}\right), \ldots,\left(r_{n}, h_{n}\right)\right)=1, \quad \text { for all } g \in \bigcap_{i=1}^{n} r_{i}^{-1} C_{H}\left(h_{i}\right) r_{i}
$$

where $\lambda_{(\mathcal{R} \times H)^{n}}$ was defined in (3.1) in function of $\lambda_{\mathcal{R} \times H}$ and $\omega$. 


\section{Chapter 4}

\section{Applications and examples}

So far, we have developed a way of understanding morphisms from the unit element to tensor products of Lagrangian algebras. In particular, we have shown a way to construct a basis for them and how the braiding acts on that basis. Let us now use that information to evaluate and generate designs for arrangements of qubits and of quantum gates.

\subsection{Computing dimensions of ground states}

As we mentioned earlier, vector spaces $\operatorname{Hom}_{\mathcal{Z}(G, \omega)}(\mathbb{C}, L[H, \gamma])$ are interpreted by physicists as ground states of a quantum mechanical system. Knowing the dimension of those spaces is useful, roughly, to determine how much information can be encoded in them. In this section, we calculate it for several cases.

\section{Central Subgroups}

Proposition 4.1.1. Let $G$ be a finite group and $L(H, \gamma)$ a Lagrangian algebra in $\mathcal{Z}\left(\operatorname{Vec}_{G}\right)$, where $H \subset G$ is a central subgroup. Then

$$
\operatorname{dim}\left(\operatorname{Hom}_{\mathcal{Z}\left(\operatorname{Vec}_{G}\right)}\left(\mathbb{C}, L(H, \gamma)^{\otimes n}\right)\right)=|G|^{n-1} .
$$

Moreover, the representation of $\mathcal{B}_{n}$ is actually a representation of the symmetric group.

Proof. Since $H$ is a central subgroup, $g \triangleright(r, h)=(g \triangleright r, h)$ and

$$
\left|\chi_{r_{1}, h_{1}} \otimes \cdots \otimes \chi_{r_{k}, h_{k}}\right|=h_{1} \cdots h_{k},
$$

for any $r_{1}, \ldots, r_{k} \in R, h_{1}, \ldots, h_{n} \in H$. Hence,

$$
\begin{aligned}
\left|(\mathcal{R} \times H)_{e}^{n}\right| & =\left|\left(\mathcal{R}^{n} / G\right)\right|\left|H^{n-1}\right| \\
& =[G: H]^{n}|H|^{n-1} \\
& =|G|^{n-1} .
\end{aligned}
$$


To determine the number of regular orbits, notice that $\epsilon: H \times H \rightarrow \mathbb{C}^{\times}$is a bicharacter such that $\epsilon\left(h_{1}, h_{2}\right) \epsilon\left(h_{2}, h_{1}\right)=1$. Then by equation (3.8) an element

$$
\left(\left(r_{1}, h_{1}\right), \ldots,\left(r_{n}, h_{n}\right)\right) \in(\mathcal{R} \times H)_{e}^{n}
$$

is regular if and only if

$$
\prod_{i=1}^{n} \epsilon\left(h, h_{i}\right)=1, \quad \text { for all } h \in H .
$$

But $\prod_{i=1}^{n} \epsilon\left(h, h_{i}\right)=\epsilon\left(h, h_{1} \cdots h_{n}\right)=\epsilon\left(h^{\prime}, e\right)=1$. Hence every element is regular. By Corollary 3.3.1 the dimension of $\operatorname{Hom}_{\mathcal{Z}\left(\operatorname{Vec}_{G}\right)}\left(\mathbb{C}, L(H, \gamma)^{\otimes n}\right)$ is $|G|^{n-1}$.

Finally, using equation (3.3), we see that

$$
\begin{aligned}
\sigma_{i}^{\prime} \circ \sigma_{i}^{\prime}\left(\chi_{r_{1}, h_{1}} \otimes \cdots \otimes \chi_{r_{n}, h_{n}}\right) & =\epsilon\left(h_{i}, h_{i+1}\right) \epsilon\left(h_{i+1}, h_{i}\right)\left(\chi_{r_{1}, h_{1}} \otimes \cdots \otimes \chi_{r_{n}, h_{n}}\right) \\
& =\chi_{r_{1}, h_{1}} \otimes \cdots \otimes \chi_{r_{n}, h_{n}} .
\end{aligned}
$$

Hence representation of $\mathcal{B}_{n}$ factors as a representation of $S_{n}$.

\section{Lagrangian algebra of the form $L(H, 1)$}

The Lagrangian algebras $L(H, 1)$ as an object in $\mathcal{Z}\left(\operatorname{Vec}_{G}\right)$ are completely determined by the crossed $G$-set $G \times_{H} H$, and the monomial representation $\operatorname{Hom}\left(\mathbb{C}, L(H, 1)^{\otimes}\right)$ is a permutation representation, see Example 3.2.1. Let us see some extreme cases:

Case $H=\{e\}$

In this case the crossed $G$-set is $G$ with the regular action and grading map the constant map $e$. It is clear that the braiding $c_{G, G}$ is just the flip map

$$
\left(g_{1}, g_{2}\right) \mapsto\left(g_{2}, g_{1}\right)
$$

hence, really the symmetric group $S_{n}$ acts on $G^{n}$.

The set of $G$-orbits is in bijection with $G^{n-1}$,

$$
\begin{aligned}
\mathcal{O}\left(G^{n}\right) & \rightarrow G^{n-1} \\
\mathcal{O}_{G}\left(g_{1}, g_{2}, \ldots, g_{n}\right) & \mapsto\left(e, g_{1}^{-1} g_{2}, \ldots, g_{1}^{-1} g_{n}\right) .
\end{aligned}
$$

Using the previous map the action of $S_{n}$ is given by

$$
\sigma_{1}\left(g_{1}, \ldots, g_{n-1}\right)=\left(g_{1}^{-1}, g_{1}^{-1} g_{2}, \ldots, g_{1}^{-1} g_{n-1}\right)
$$

and

$$
\sigma_{i}\left(g_{1}, \ldots, g_{i}, g_{i+1}, \ldots, g_{n-1}\right)=\left(g_{1}, \ldots, g_{i+1}, g_{i}, \ldots, g_{n-1}\right), \quad 1<i<n .
$$

It is clear that permutation action of $S_{n}$ on $G^{n-1}$ is faithful, thus the image is isomorphic to $S_{n}$. 
Case $H=G$

In this case the crossed $G$-set is $G$ with the action by conjugation and grading map the identity map. Hence, the braiding is given by

$$
c_{G, G}:(x, y) \mapsto\left(y, y^{-1} x y\right)
$$

Note $c_{G, G}$ is symmetric if and only if $G$ is abelian.

If $G$ is abelian, $G_{e}^{n}=\left\{\left(g_{1}, \ldots, g_{n-1},\left(g_{1} \ldots g_{n-1}\right)^{-1}\right)\right\}$ is the set of orbits and as the previous example the group $S_{n}$ acts faithfully.

\section{Dihedral group}

If we take $H$ to be a normal subgroup of $G$, the following proposition provides a way to simplify the situation.

Proposition 4.1.2. Let $G$ be a finite group, $H \unlhd G, \mathcal{R}$ a collection of representatives for $G / H$. Define $B_{\gamma}[H] \in \mathcal{Z}\left(\operatorname{Vec}_{G}\right)$ as

$$
B(H, \gamma):=\operatorname{span}\left\{b_{r, h} \mid r \in \mathcal{R}, h \in H\right\}
$$

with grading $\left|b_{r, h}\right|=h$ and the $G$-action

$$
g \triangleright b_{r, h}=\epsilon\left(\kappa(g, r)^{r^{-1}} h\right) b_{g \triangleright r,{ }^{g} h} .
$$

Then, the mapping

$$
\begin{aligned}
B(H, \gamma) & \rightarrow L(H, \gamma) \\
b_{r, h} & \mapsto \chi_{r, r^{-1} h}
\end{aligned}
$$

is an isomorphism in $\mathcal{Z}(G, \omega)$.

Proof. We need to show the map preserves the grading and the $G$-representation. We have

$$
\left|\chi_{r, r^{-1}}\right|={ }^{r}\left(r^{r^{-1}} h\right)=h=\left|b_{r, h}\right|
$$

Now, since

$$
g \cdot \chi_{r, r^{-1} h}=\epsilon\left(\kappa(g, r),{ }^{-1} h\right) \chi_{g \triangleright r,{ }^{\kappa(g, r)}\left(r^{-1} h\right)},
$$

and

$$
{ }^{\kappa(g, r)}\left({ }^{-1} h\right)={ }^{(g \triangleright r)^{-1}} g h g^{-1},
$$

we have that

$$
g \triangleright b_{r, h}=\epsilon\left(\kappa(g, r),{ }^{r^{-1}} h\right) a_{g \triangleright r,(g \triangleright r)^{-1}\left({ }^{g} h\right)} .
$$

Hence by (4.1) the map is equivariant. 
Proposition 4.1.2 works particularly well when $\gamma=1$, since equation (4.1) is just

$$
g \triangleright b_{r, h}=b_{g \triangleright r, g h} .
$$

Thus, the action of $G$ is understood as separate, independent actions on each label. We use this idea in the following example.

Let $G=D_{2 k}$ be the dihedral groups of order $2 k$ and $H=\langle r\rangle$. We take $\mathcal{R}=\{e, s\}=$

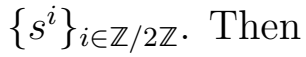

$$
\left|b_{s^{i_{1}, r^{j_{1}}}} \otimes \cdots \otimes b_{s^{i_{n}, r^{j_{n}}}}\right|=r^{\sum_{m=1}^{n} j_{m}},
$$

and

$$
\operatorname{dim}\left(B(H, \gamma)_{e}^{\otimes n}\right)=2^{n} \times k^{n-1} .
$$

Since

$$
\left(s^{i} r^{j}\right)\left(s^{k}\right)=s^{i+k} r^{(-1)^{k} j}
$$

we have that

$$
\left(s^{i} r^{j}\right) \triangleright s^{k}=s^{i+k}, \quad \quad \text { and } \quad \kappa\left(s^{i} r^{j}, s^{k}\right)=r^{(-1)^{k} j} .
$$

Hence, the action, on the set label is

$$
s^{i} r^{j}\left(s^{k}, r^{l}\right)=\left(s^{i+k}, r^{l}\right)
$$

It follows that the number of orbits in $(\mathcal{R} \times H)_{e}^{n}$ is

$$
2^{n-1} \times k^{n-1}=|G|^{n-1} .
$$

Since $\gamma=1$ all orbits are regular and then $\operatorname{dim}\left(\operatorname{Hom}_{\mathcal{Z}(G, \omega)}\left(\mathbb{C}, L(H, 1)^{\otimes n}\right)\right)=|G|^{n-1}$.

\subsection{Quantum Gates}

Since spaces of the form $\operatorname{Hom}_{\mathcal{Z}(G, \omega)}(\mathbb{C}, L[H, \gamma])$ are interpreted by physicists as means to store information, transformations on this space would then implement operations on this information. We intend to see what operations can be achieved using the representation of $\mathcal{B}_{n}$. Of particular interest are entagling gates, which we now discuss.

\section{Entangling Gates}

In the following, let $V, W$ be complex vector spaces of dimension $n$ and $m$ respectively. Suppose, without loss of generality, that $n \leq m$. We will merely enunciate some classic results, whose proofs can be found in [19].

Definition 4.2.1. A vector $\psi \in V \otimes W$ said to be separable if there exist vectors $v \in V$ and $w \in W$ such that $\psi=v \otimes w$. 
Definition 4.2.2. An operator, $T \in \operatorname{End}_{\mathbb{C}}(V \otimes W)$ is said to be entangling if there exist a separable vector $\psi \in V \otimes W$ such that $T \psi$ is not separable.

Example 4.2.1. Consider $V=W=\mathbb{C}^{2}$ with basis $\beta=\{|0\rangle,|1\rangle\}$. Define the operator

$$
U|m\rangle \otimes|n\rangle=|m\rangle \otimes|m+n\rangle
$$

where $m, n \in\{0,1\}$ and the sum on the right hand side is taken modulo 2. This is called the CNOT gate. Notice that

$$
U\left(\frac{1}{\sqrt{2}}(|0\rangle+|1\rangle) \otimes|0\rangle\right)=\frac{1}{\sqrt{2}}(|0\rangle \otimes|0\rangle+|1\rangle \otimes|1\rangle)
$$

So CNOT is entangling.

Let $H$ be a subgroup of $G$ (the group we fixed from the first chapter) and $\gamma \in C^{2}\left(H, \mathbb{C}^{\times}\right)$ such that $d^{3}(\gamma)=\left.\omega\right|_{H}$ ( $\omega$ is the 3-cocycle fixed from the first chapter). In the category $\mathcal{Z}(G, \omega)$, consider the Lagrangian algebra $L[H, \gamma]$ and set

$$
\mathcal{H}_{4}=\operatorname{Hom}_{\mathcal{Z}(G, \omega)}\left(\mathbb{1}, L[H, \gamma]^{\otimes 4}\right)
$$

In [5, Page 101], Cong, Cheng and Wang posed the following question:

Question 4.2.1. Does there exist a subspace $W$ of $\mathcal{H}_{4}$, a vector space $\mathcal{H}$ and an isomorphim $T: W \rightarrow \mathcal{H} \otimes \mathcal{H}$ such that the image of $\mathcal{B}_{n}$ preserves $W$ and, through $T$, implements entangling gates?

We will see in the following that the answer is on the affirmative.

Throughout this section, we will fix $G=S_{3}$, which we will understand as the dihedral group of order 6 , with generators $r, s$ subject to relations $r^{3}=s^{2}=(r s)^{2}=e$. We will also fix $\omega=1, H=\{e, s\}$ and $\gamma=1$.

First order of business is to say a couple of things about the category $\mathcal{Z}(G, 1)$. As we mentioned, simple objects of this category can be parametrized by conjugation classes, $C$ and irreducible representations, $\pi$, of their centralizers $E(C)$, all up to conjugation. In this case, this gives rise to eight simple objects, as follows:

- $C_{1}=\{e\}, E\left(C_{1}\right)=S_{3}$. This has three irreducible representations: trivial, sign and alternating, which we will denote by $A, B$ and $C$ respectively.

- $C_{2}=\left\{s, r s, r^{2} s\right\}, E\left(C_{2}\right)=\{e, s\} \cong \mathbb{Z} / 2 \mathbb{Z}$. We have two representations: trivial and sign, $D$ and $E$.

- $C_{3}=\left\{r, r^{2}\right\}, E\left(C_{3}\right)=\left\{e, r, r^{2}\right\} \cong \mathbb{Z} / 3 \mathbb{Z}$. This has three representations, which depend on the image of $r$. The trivial one, $r \mapsto 1(F) ; r \mapsto \zeta(G)$ and $r \mapsto \zeta^{2}(H)$. Here $\zeta=e^{2 \pi i / 3}$. 
The fusion rules that will be relevant most relevant to us are

$$
\begin{aligned}
& C \otimes C=A \oplus B \oplus C \\
& C \otimes D=D \oplus E \\
& D \otimes D=A \oplus C \oplus F \oplus G \oplus H
\end{aligned}
$$

With respect to the Lagrangian algebra $L[H, 1]$ (just $L$ from now on), we know that is it spanned by basis elements $\left\{\chi_{r^{m}, s^{n}}\right\}_{m=0,1,2 ; n=0,1}$. It decomposes in simple objects as $L=A \oplus C \oplus D$, with

$$
\begin{aligned}
& A=\operatorname{span}\left\{\chi_{e, e}+\chi_{r, e}+\chi_{e, s}\right\} \\
& C=\operatorname{span}\left\{2 \chi_{e, e}-\chi_{r, e}-\chi_{r^{2}, e}, \chi_{r, e}-\chi_{r^{2}, e}\right\} \\
& D=\operatorname{span}\left\{\chi_{e, s}, \chi_{r, s}, \chi_{r^{2}, e}\right\} .
\end{aligned}
$$

Example 4.2.2. Let us first consider a simple scenario: $\mathcal{H}_{2}=\operatorname{Hom}_{\mathcal{Z}(G)}\left(\mathbb{1}, L^{\otimes 2}\right) \cong\left(L^{\otimes 2}\right)_{e}^{G}$ (In general, we will write $\mathcal{H}_{n}$ for $\left(L^{\otimes n}\right)_{e}^{G}$ ). Theorem 3.3.1 gives us a recipe for constructing a basis for this space. In this case, three elements of $X_{e}^{2}$ are $((e, e),(e, e),((e, e),(e, r))$ and $((e, s),(e, s))$ whose orbits can be seen to include all of $X_{e}^{2}$. Thus, $\operatorname{dim}\left(\mathcal{H}_{2}\right)=3$, with basis

$$
\begin{aligned}
\psi_{e e, e e}= & \chi_{e, e} \otimes \chi_{e, e}+\chi_{r, e} \otimes \chi_{r, e}+\chi_{r^{2}, e} \otimes \chi_{r^{2}, e} \\
\psi_{e e, r e}= & \chi_{e, e} \otimes \chi_{r, e}+\chi_{r, e} \otimes \chi_{r^{2}, e}+\chi_{r^{2}, e} \otimes \chi_{e, e} \\
& \quad+\chi_{e, e} \otimes \chi_{r^{2}, e}+\chi_{r, e} \otimes \chi_{e, e}+\chi_{r^{2}, e} \otimes \chi_{r, e} \\
\psi_{e s, e s}= & \chi_{e, s} \otimes \chi_{e, s}+\chi_{r, s} \otimes \chi_{r, s}+\chi_{r^{2}, e} \otimes \chi_{r^{2}, e}
\end{aligned}
$$

However, there is another basis of interest, which we now construct. Its objects correspond to the images of the morphisms $\mathbb{1} \rightarrow A \otimes A \rightarrow L \otimes L, \mathbb{1} \rightarrow C \otimes C \rightarrow L \otimes L$ and $\mathbb{1} \rightarrow D \otimes D \rightarrow$ $L \otimes L$, respectively:

$$
\begin{aligned}
& \psi_{A}=\left(\chi_{e, e}+\chi_{r, e}+\chi_{e, s}\right) \otimes\left(\chi_{e, e}+\chi_{r, e}+\chi_{e, s}\right) \\
& \psi_{C}=\operatorname{Av}_{G}\left(\left(2 \chi_{e, e}-\chi_{r, e}-\chi_{s, e}\right) \otimes\left(2 \chi_{e, e}-\chi_{r, e}-\chi_{r^{2}, e}\right)\right) \\
& \psi_{D}=\operatorname{Av}_{G}\left(\chi_{e, s} \otimes \chi_{e, s}\right) .
\end{aligned}
$$

The previous example shows how to encode a qutrit. However, we need more than one in order to implement entangling gates. There is no general construction to encode qubits inside spaces of the form $\mathcal{H}_{n}$. There are some ad hoc examples in the literature. For instance, the following example was proposed in [5, Section 5.6.6].

Example 4.2.3. Define $\mathcal{H} \subset \mathcal{H}_{4}$ to be the image of the inclusion $\mathcal{H}_{2} \otimes \mathcal{H}_{2} \hookrightarrow \mathcal{H}_{4}$. This subspace comes already with the structure of tensor product and encodes two qutrits. In [5], the question of what gates do images of $\mathcal{B}_{n}$ encode was left open. Let us show that this example does not produce any interesting gates. 
First, notice that generators $\sigma_{1}$ and $\sigma_{3}$ produce gates of the form $T \otimes$ id and id $\otimes T$, so they are of no interest. Let us focus then on $\sigma_{2}$.

It turns out that $\sigma_{2}$ does not preserve $\mathcal{H}$. For example, we have

$$
\begin{aligned}
& \sigma_{2}^{\prime}\left(\psi_{1} \otimes \psi_{3}\right)=\chi_{e, e} \otimes \chi_{e, s} \otimes \chi_{e, e} \otimes \chi_{e, s}+\chi_{e, e} \otimes \chi_{r, s} \otimes \chi_{e, e} \otimes \chi_{r, s}+\chi_{e, e} \otimes \chi_{r^{2}, s} \otimes \chi_{e, e} \otimes \chi_{r^{2}, s} \\
& \quad+\chi_{r, e} \otimes \chi_{e, s} \otimes \chi_{r, e} \otimes \chi_{e, s}+\chi_{r, e} \otimes \chi_{r, s} \otimes \chi_{r, e} \otimes \chi_{r, s}+\chi_{r, e} \otimes \chi_{r^{2}, e} \otimes \chi_{r, e} \otimes \chi_{r^{2}, e} \\
& \quad+\chi_{r^{2}, e} \otimes \chi_{e, s} \otimes \chi_{r^{2}, e} \otimes \chi_{e, s}+\chi_{r^{2}, e} \otimes \chi_{r, s} \otimes \chi_{r^{2}, e} \otimes \chi_{r, s}+\chi_{r^{2}, e} \otimes \chi_{r^{2}, e} \otimes \chi_{r^{2}, e} \otimes \chi_{r^{2}, s}
\end{aligned}
$$

In the first summand, the degree of the first two factors is $s$. Thus, it does not correspond to an element of $\mathcal{H}_{2}$. We conclude that $\sigma_{2}\left(\psi_{1} \otimes \psi_{3}\right)$ does not belong to $\mathcal{H}$.

The only hope remaining would be to consider the representation of the pure braid group, $\mathcal{P}_{n}$. However, it can be checked that $\left.\left(\sigma_{2}^{\prime}\right)^{2}\right|_{\mathcal{H}}=1$.

Example 4.2.4. We propose a new way to encode two qubits. Let $\mathcal{H}$ be the subspace of $\mathcal{H}_{5}$ such that objects in $\mathcal{H}$ factor through the inclusion $D \otimes C \otimes C \otimes C \otimes D \hookrightarrow L^{\otimes 5}$.

Proposition 4.2.1. The dimension of $\mathcal{H}$ is four.

Proof. Working out the fusion rules, we get

$D \otimes C \otimes C \otimes C \otimes D=2(C \oplus A \oplus B \oplus C \oplus G \oplus H \oplus F \oplus H \oplus F \oplus G \oplus C$

$$
\oplus A \oplus B \oplus C \oplus G \oplus H \oplus F \oplus H \oplus F \oplus G)
$$

From which we deduce that there are four copies of $A$ in $D \otimes C \otimes C \otimes C \otimes D$.

The subspace $\mathcal{H}$ encodes 2 qubits. They correspond to morphisms:

$$
\begin{aligned}
& |E E\rangle: \mathbb{1} \rightarrow E \otimes E \rightarrow(D \otimes C) \otimes(C \otimes E) \rightarrow D \otimes C \otimes C \otimes C \otimes D \\
& |E D\rangle: \mathbb{1} \rightarrow E \otimes E \rightarrow(D \otimes C) \otimes(C \otimes D) \rightarrow D \otimes C \otimes C \otimes C \otimes D \\
& |D E\rangle: \mathbb{1} \rightarrow D \otimes D \rightarrow(D \otimes C) \otimes(C \otimes E) \rightarrow D \otimes C \otimes C \otimes C \otimes D \\
& |D D\rangle: \mathbb{1} \rightarrow D \otimes D \rightarrow(D \otimes C) \otimes(C \otimes D) \rightarrow D \otimes C \otimes C \otimes C \otimes D
\end{aligned}
$$

Images of these morphisms are spans of the following vectors, respectively:

$$
\begin{aligned}
& \psi_{D D}=\operatorname{Av}_{G}\left[\left(2 \chi_{e, e}-\chi_{r, e}-\chi_{r^{2}, e}\right) \chi_{e, s}\left(2 \chi_{e, e}-\chi_{r, e}-\chi_{r^{2}, e}\right)\left(2 \chi_{e, e}-\chi_{r, e}-\chi_{r^{2}, e}\right) \chi_{e, s}\right] \\
& \psi_{D E}=\operatorname{Av}_{G}\left[\left(2 \chi_{e, e}-\chi_{r, e}-\chi_{r^{2}, e}\right) \chi_{e, s}\left(\chi_{r^{2}, e}-\chi_{e, e}\right)\left(\chi_{r^{2}, e}-\chi_{e, e}\right) \chi_{r, s}\right] \\
& \psi_{E D}=\operatorname{Av}_{G}\left[\left(\chi_{r, e}-\chi_{r^{2}, e}\right) \chi_{e, s}\left(2 \chi_{r, e}-\chi_{r^{2}, e}-\chi_{e, e}\right)\left(2 \chi_{r, e}-\chi_{r^{2}, e}-\chi_{e, e}\right) \chi_{r, s}\right] \\
& \psi_{E E}=\operatorname{Av}_{G}\left[\left(\chi_{r, e}-\chi_{r^{2}, e}\right) \chi_{e, s}\left(2 \chi_{e, e}-\chi_{r, e}-\chi_{r^{2}, e}\right)\left(\chi_{r, e}-\chi_{r^{2}, e}\right) \chi_{e, s}\right]
\end{aligned}
$$


Given the symmetries in the definition of $\mathcal{H}$, it is to be expected that it is not preserved under the full action of $\mathcal{B}_{n}$ but rather under the subgroup

$$
K=\left\langle\sigma_{2}, \sigma_{3}, \sigma_{1} \sigma_{2} \sigma_{3} \sigma_{4} \sigma_{3} \sigma_{2} \sigma_{1}\right\rangle .
$$

We can explicitly calculate the images of these elements. In particular $\sigma_{2}$ acts as

$$
\begin{aligned}
|D D\rangle & \mapsto|D D\rangle \\
|D E\rangle & \mapsto|D E\rangle \\
|E D\rangle & \mapsto|E E\rangle \\
|E E\rangle & \mapsto|E D\rangle
\end{aligned}
$$

Thus, $\left.\sigma_{2}^{\prime}\right|_{\mathcal{H}}$ can be seen as a CNOT gate.

Example 4.2.5. The previous example can be generalized at once to make as many qubits as wanted. For instance, if we want to encode 3 qubits we take $\mathcal{H}_{6}$ and consider morphisms that factor through the inclusion $D \otimes(C)^{\otimes 4} \otimes D \hookrightarrow L^{\otimes 6}$. A basis will now be indexed by triples in $\{D, E\}^{3}$. Action of the generators is summarized in Table 4.2.5. We see that $\sigma_{2}$ acts as CNOT $\otimes \mathrm{id}$, with the second qubit as control. The generator $\sigma_{4}$ is id $\otimes$ CNOT with the second qubit as control as well. Finally, $\sigma_{3}$ is also a controlled NOT, the control qubit being the sum of the first and the third one.

\begin{tabular}{l|c|c|c} 
& $\sigma_{2}$ & $\sigma_{3}$ & $\sigma_{4}$ \\
\hline DDD & DDD & DDD & DDD \\
DDE & DDE & DEE & DDE \\
DED & EED & DED & DEE \\
DEE & EEE & DDE & DED \\
EDD & EDD & EED & EDD \\
EDE & EDE & EDE & EDE \\
EED & DED & EDD & EEE \\
EEE & DEE & EEE & EED
\end{tabular}

Table 4.1: Action of Generators on the three qubits 


\section{Bibliography}

[1] Bojko Bakalov and Alexander Kirillov Jr. Lectures on tensor categories and modular functors. Vol. 21. University Lecture Series. American Mathematical Society, Providence, RI, 2001, pp. x+221. ISBN: 0-8218-2686-7.

[2] Salman Beigi, Peter W. Shor, and Daniel Whalen. "The quantum double model with boundary: condensations and symmetries". In: Comm. Math. Phys. 306.3 (2011), pp. 663694. ISSN: 0010-3616. DOI: 10.1007/s00220-011-1294-x. URL: http://dx.doi.org/ $10.1007 / \mathrm{s} 00220-011-1294-\mathrm{x}$.

[3] Hector Bombin and MA Martin-Delgado. "Family of non-Abelian Kitaev models on a lattice: Topological condensation and confinement". In: Physical Review B 78.11 (2008), p. 115421.

[4] Sergey B Bravyi and A Yu Kitaev. "Quantum codes on a lattice with boundary". In: arXiv preprint quant-ph/9811052 (1998).

[5] Iris Cong, Meng Cheng, and Zhenghan Wang. "Topological Quantum Computation with Gapped Boundaries". In: arXiv preprint arXiv:1609.02037 (2016).

[6] Alexei Davydov and Darren Simmons. "On Lagrangian algebras in group-theoretical braided fusion categories". In: Journal of Algebra 471 (2017), pp. 149-175. ISSN: 00218693. DOI: http://dx . doi .org/10.1016/j . jalgebra. 2016.09.016. URL: http: //www.sciencedirect.com/science/article/pii/S0021869316303246.

[7] David Deutsch. "Quantum theory, the Church-Turing principle and the universal quantum computer". In: Proceedings of the Royal Society of London A: Mathematical, Physical and Engineering Sciences. Vol. 400. 1818. The Royal Society. 1985, pp. 97-117.

[8] Nicolás Escobar-Velásquez, César Galindo, and Zhenghan Wang. "Braid Group Representations from Braiding Gapped Boundaries of Dijkgraaf-Witten Theories". In: arXiv preprint arXiv:170\%.03884 (2017).

[9] Pavel I Etingof et al. Tensor categories. Vol. 205. American Mathematical Society Providence, RI, 2015.

[10] Pavel Etingof, Eric Rowell, and Sarah Witherspoon. "Braid group representations from twisted quantum doubles of finite groups". In: Pacific journal of mathematics 234.1 (2008), pp. 33-41. 
[11] Pavel Etingof et al. Tensor categories. Vol. 205. Mathematical Surveys and Monographs. American Mathematical Society, Providence, RI, 2015, pp. xvi+343. ISBN: 978-1-4704-2024-6. DOI: 10.1090/surv/205. URL: http://dx . doi .org/10 . 1090/ surv/205.

[12] Richard P Feynman. "Simulating physics with computers". In: International journal of theoretical physics 21.6 (1982), pp. 467-488.

[13] Lov K Grover. "A fast quantum mechanical algorithm for database search". In: Proceedings of the twenty-eighth annual ACM symposium on Theory of computing. ACM. 1996, pp. 212-219.

[14] André Joyal and Ross Street. "Braided tensor categories". In: Advances in Mathematics 102.1 (1993), pp. 20-78.

[15] Gregory Karpilovsky. Projective representations of finite groups. Vol. 94. Monographs and Textbooks in Pure and Applied Mathematics. Marcel Dekker, Inc., New York, 1985, pp. xiii+644. ISBN: 0-8247-7313-6.

[16] Christian Kassel. Quantum groups. Vol. 155. Springer Science \& Business Media, 2012.

[17] A.Yu. Kitaev. "Fault-tolerant quantum computation by anyons". In: Annals of Physics 303.1 (2003), pp. 2-30. ISSN: 0003-4916. DOI: http://dx.doi.org/10.1016/S00034916 (02) 00018-0. URL: http://www. sciencedirect.com/science/article/pii/ S0003491602000180.

[18] Saunders Mac Lane. Categories for the working mathematician. Vol. 5. Springer Science \& Business Media, 2013.

[19] Michael A Nielsen and Isaac Chuang. Quantum computation and quantum information. AAPT, 2002.

[20] Peter W Shor. "Polynomial-time algorithms for prime factorization and discrete logarithms on a quantum computer". In: SIAM review 41.2 (1999), pp. 303-332.

[21] Vladimir G Turaev. Quantum invariants of knots and 3-manifolds. Vol. 18. Walter de Gruyter GmbH \& Co KG, 2016.

[22] John HC Whitehead. "Combinatorial homotopy. II". In: Bulletin of the American Mathematical Society 55.5 (1949), pp. 453-496. 